\title{
ON THE RELATIVE CONSISTENCY STRENGTH OF DETERMINACY HYPOTHESES
}

BY

\author{
ALEXANDER S. KECHRIS ${ }^{1}$ AND ROBERT M. SOLOVAY ${ }^{2}$
}

\begin{abstract}
For any collection of sets of reals $C$, let $C$-DET be the statement that all sets of reals in $C$ are determined. In this paper we study questions of the form: For given $C \subseteq C^{\prime}$, when is $C^{\prime}$-DET equivalent, equiconsistent or strictly stronger in consistency strength than $C$-DET (modulo ZFC)? We focus especially on classes $C$ contained in the projective sets.
\end{abstract}

\section{Introduction.}

1.1. For any set $A \subseteq \omega^{\omega}=\mathbf{R}$ (= the set of "reals"), consider the associated infinite game $G_{A}$ :

$\begin{array}{llll}\text { I } & \alpha(0) & \alpha(2) & \\ \text { II } & & \alpha(1) & \alpha(3)\end{array}$

in which player I wins if $\alpha \in A$, and II wins otherwise. We call $A$ determined if the corresponding game $G_{A}$ is determined, i.e. if either player I or player II has a winning strategy. For any class of sets $\mathscr{C}$ we abbreviate by $\mathscr{C}$-DET the statement that every set of reals $A \in \mathscr{C}$ is determined. The Definable Determinacy Hypothesis is the assertion that every set of reals which is ordinal-definable from a countable sequence of ordinals is determined, i.e. $\mathscr{C}$-DET for $\mathscr{C}=\mathrm{OD}\left(\mathrm{ORD}^{\omega}\right)$, where ORD $=$ the class of ordinals. This hypothesis is being considered as a plausible candidate for a strong set theoretical axiom transcending the limits of classical ZFC set theory. With this motivation an extensive study has been made over the last two decades of its consequences for the structure theory of the continuum, its relationships to other proposed strong axioms of set theory, particularly large cardinal hypotheses, and its logical strength.

The Definable Determinacy Hypothesis naturally ramifies in a (nonnecessarily strict) hierarchy of hypotheses $\mathscr{C}$-DET for various $\mathscr{C}$ contained in $\mathrm{OD}\left(\mathrm{ORD}^{\omega}\right)$. Our main concern in this paper is the analysis of this hierarchy, particularly focusing on its lower levels, where the classes $\mathscr{C}$ contain only projective sets of reals. Typical questions we will be studying are of the form: For given $\mathscr{C} \subseteq \mathscr{C}^{\prime}$ as above, when is $\mathscr{C}^{\prime}$-DET equivalent, equiconsistent or strictly stronger in consistency strength than

Received by the editors July 6, 1984.

1980 Mathematics Subject Classification. Primary 02K30, 02 K05.

${ }^{1}$ Partially supported by NSF Grant MCS 79-20465 and an A. P. Sloan Foundation Fellowship.

${ }^{2}$ Partially supported by NSF Grant MCS 79-06077. 
$\mathscr{C}$-DET (modulo ZFC)? We think that such a logical analysis is helpful in providing new insights on the nature and the plausibility of consistency of this powerful hypothesis.

1.2. To put things in perspective, we will review first some known results on the relative consistency strength of determinacy hypotheses. It is convenient to summarize this information in the form of a table. The first column lists progressively stronger (not necessarily strictly) forms of determinacy relative to the base theory ZFC. The notation $\mathscr{C}$-DET $\Leftrightarrow \mathscr{C}^{\prime}$-DET means therefore that

$$
\mathrm{ZFC} \vDash \mathscr{C} \text {-DET } \Leftrightarrow \mathscr{C}^{\prime} \text {-DET, }
$$

while the notation $\mathscr{C}$-DET $<\mathscr{C}^{\prime}$-DET means that $\mathscr{C}^{\prime}$-DET is consistencywise stronger than $\mathscr{C}$-DET, i.e.

$$
\mathrm{ZFC}+\mathscr{C}^{\prime}-\mathrm{DET} \vDash \operatorname{Con}(\mathrm{ZFC}+\mathscr{C} \text {-DET }) .
$$

Finally $\mathscr{C}$-DET $\approx \mathscr{C}^{\prime}$-DET means that ZFC $+\mathscr{C}$-DET is equiconsistent with ZFC + $\mathscr{C}^{\prime}$-DET. The notation for the various classes used is standard, but we explain it anyway after the table.

The second column in the table lists large cardinal hypotheses known to have some connections with the corresponding determinacy hypotheses appearing in the same row. (Our notational conventions for $\Leftrightarrow$ and $<$ apply here as well.) This provides a calibration of the strength of the corresponding determinacy hypotheses in the scale of large cardinal axioms (see [KM]).

Following the table we also give the appropriate references for the results contained in it.

As usual $\Sigma_{n}^{1}, \Pi_{n}^{1}, \Delta_{n}^{1}$ denote the standard projective pointclasses (see [Mo1]). The pointclass $\xi-\Pi_{1}^{1}$ for $\xi<\omega_{1}$ is defined as follows:

$$
\begin{aligned}
A \in \xi-\Pi_{1}^{1} \Leftrightarrow \quad & \text { There is a sequence } \\
& A_{0}, A_{1}, \ldots, A_{\eta}, \ldots(\eta<\xi) \text { of } \Pi_{1}^{1} \\
& \text { sets such that setting } \\
& A_{\xi}=\varnothing \text { we have: } \\
& x \in A \Leftrightarrow \text { least } \eta \leqslant \xi \text { with } x \notin A_{\eta} \text { is odd. }
\end{aligned}
$$

The pointclass $\Sigma_{1}^{0}\left(\Pi_{1}^{1}\right)$ consists of all countable unions of boolean combinations of $\Pi_{1}^{1}$ sets (similarly we can define $\Sigma_{\xi}^{0}\left(\Pi_{1}^{1}\right)$ for $\left.\xi<\omega_{1}\right)$. By $\mathscr{A}\left(\Pi_{1}^{1}\right)$ we denote the pointclass of all sets obtained by applying the classical operation $\mathscr{A}$ (see [Mo1]) to $\Pi_{1}^{1}$ sets.

Beyond the projective level, the pointclass HYP consists of all hyperprojective sets and the pointclass IND of all the inductive sets of reals (see [Mo1]). Finally $L(\mathbf{R})$ is the smallest inner model of ZF containing all the reals.

From the results in this table, (1) follows from combined work of Martin [Ma1] and Harrington [H], while (2) is due to Martin [Ma2]. Further (3), (4) are proved in Simms [Si]. For an extensive study of the relationship of various forms of $\Gamma$-DET, for $\Gamma$ a pointclass at low levels of $\Delta_{2}^{1}$ and the theory of Mitchell models [Mi1, Mi2], see Steel [St1]. Theorem (5) and (6) are due to Martin [Ma2, Ma3]. 


\section{TABLE 1}

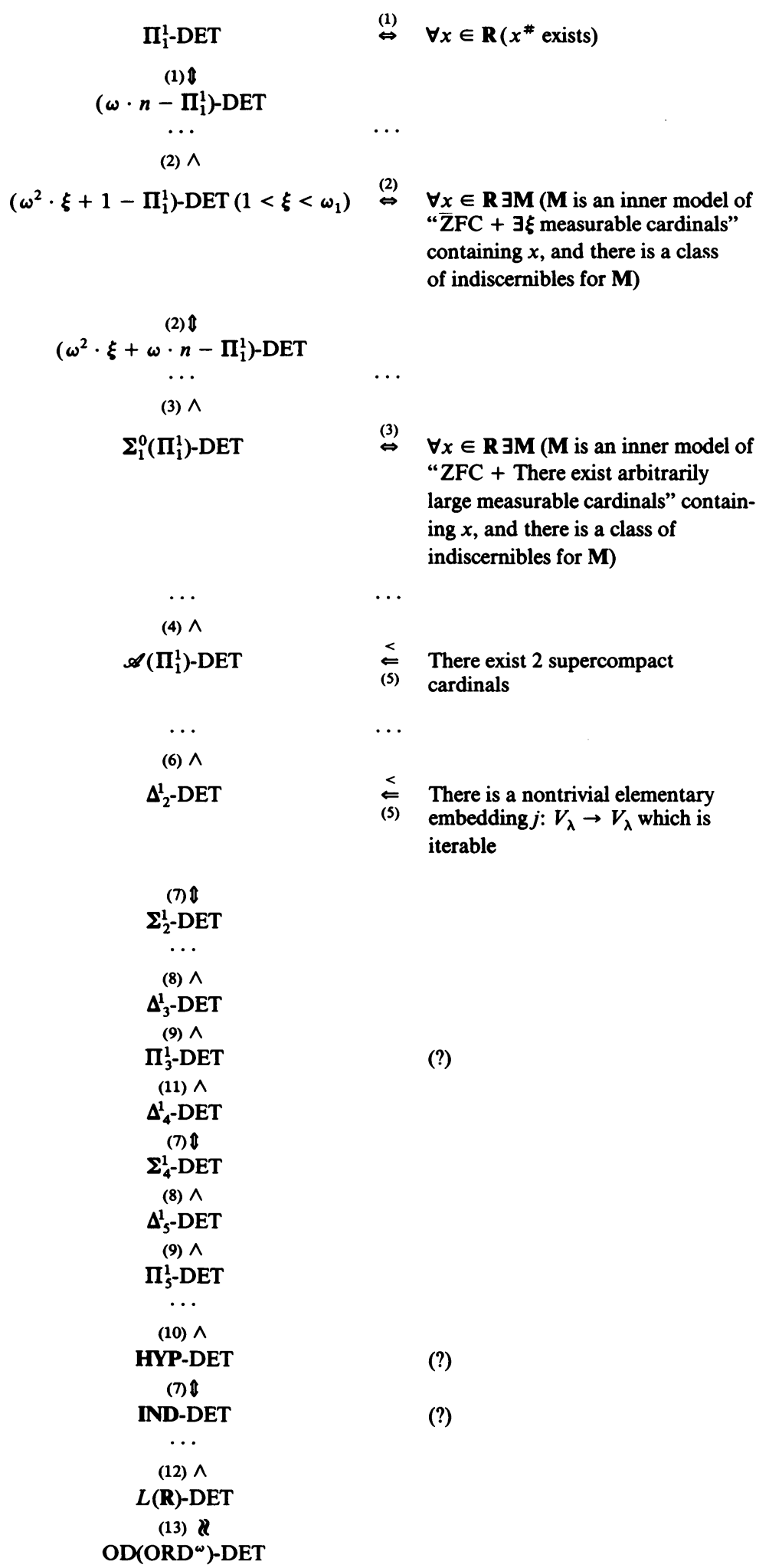


The equivalences (7) are due to Martin [Ma4]. In $\$ 5$ (Theorem 5.1) we give a simple new proof of a more general result including these theorems. The strict relations (8) were proved by Martin [Ma5], while (9) are due to Kechris and Moschovakis (see [KeM]). Moschovakis proved (10) (see [KeM]) and (12) (see [Mo2]), while it is not clear who first proved (11).

The last equiconsistency theorem (13), a folklore result, is actually only one part of the following:

THEOREM (FOLKLORE). The following theories are equiconsistent:

(i) $\mathrm{ZFC}+\mathrm{OD}\left(\mathrm{ORD}^{\omega}\right)-\mathrm{DET}$.

(ii) $\mathrm{ZFC}+L(\mathbf{R})-\mathrm{DET}$.

(iii) $\mathrm{ZF}+\mathrm{DC}+L(\mathbf{R})-\mathrm{DET}$.

(iv) $\mathrm{ZF}+\mathrm{DC}+\mathrm{AD}+V=L(\mathbf{R})$.

(v) $\mathrm{ZF}+\mathrm{DC}+\mathrm{AD}$.

Here AD (the "Axiom of Determinacy") is the assertion power(R)-DET. Actually by a recent result of Kechris [Ke1]

$$
\mathrm{ZF}+\mathrm{AD}+V=L(\mathbf{R}) \Rightarrow \mathrm{DC} .
$$

Thus one can add to (i)-(v),

(vi) $\mathrm{ZF}+\mathrm{AD}+V=L(\mathbf{R})$.

(vii) $\mathrm{ZF}+L(\mathbf{R})-\mathrm{DET}$.

(viii) $\mathrm{ZF}+\mathrm{AD}$.

A brief comment about the proof of this theorem: Clearly (i) $\Rightarrow$ (ii) $\Rightarrow$ (iii) and (iii), (iv), (v) are equiconsistent, since $\mathrm{ZF}+\mathrm{DC} \Rightarrow L(\mathbf{R}) \vDash \mathrm{DC}$. Finally if $\mathrm{ZF}+\mathrm{DC}$ $+\mathrm{AD}+V=L(\mathbf{R})$ holds, then in the generic extension of the universe obtained by forcing with the set of conditions $\bigcup_{\xi<\omega_{1}} \mathbf{R}^{\xi}$ (i.e. all countable wellordered sequences of reals), we have that $\mathrm{ZFC}+\mathrm{OD}\left(\mathrm{ORD}^{\omega}\right)$-DET holds.

Notice in the preceding theorem the consistencywise reduction of the maximal determinacy hypothesis $\mathrm{OD}\left(\mathrm{ORD}^{\omega}\right)$-DET to the primafacie weaker one $L(\mathbf{R})$-DET. Is any further reduction possible? The following result provides a positive answer.

THEOREM 2.1. ZFC $+\mathrm{DC} \vdash\left(\Delta_{1}^{2}\right)^{L(\mathbf{R})}$-DET $\Rightarrow L(\mathbf{R})$-DET.

Thus the preceding list of equiconsistencies can be extended by

(ix) $\mathrm{ZF}+\mathrm{DC}+\left(\Delta_{1}^{2}\right)^{L(\mathbf{R})}-\mathrm{DET}$,

(x) $\mathrm{ZFC}+\left(\Delta_{1}^{2}\right)^{L(\mathbf{R})}-\mathrm{DET}$.

Here of course $\left(\Delta_{1}^{2}\right)^{L(\mathbf{R})}$ is the class of all sets of reals which are $\Delta_{1}^{2}$ in $L(\mathbf{R})$.

This reduction from $L(\mathbf{R})$-DET to just $\left(\Delta_{1}^{2}\right)^{L(\mathbf{R})}$-DET could be of some importance in attempts to prove the consistency of the Definable Determinacy Hypothesis from, say, large cardinal hypotheses, since one would only have to deal with sets which are $\left(\Delta_{1}^{2}\right)^{L(\mathbf{R})}$. By the results of Martin and Steel (see [MMS and MSt]) these sets admit a very nice normal form, namely $\left(\Sigma_{1}^{2}\right)^{L(\mathbf{R})}=\supset^{R} \Pi_{1}^{1}$, where $\supset^{\mathbf{R}}$ is the real game quantifier, so they may be amenable to some kind of analysis.

Modulo a small improvement, which we will now explain, the reduction to $\left(\Delta_{1}^{2}\right)^{L(\mathbf{R})}$-DET is best possible. 
Let $\mathrm{ZF}_{N}$ be (ZF-Replacement-Separation) $+\Sigma_{N}$-Replacement $+\Sigma_{N}$ Separation for $N=1,2, \ldots$. The class $\mathrm{PR}_{N}\left(\Delta_{1}^{2}\right)^{L(\mathbf{R})}$, of provable in $\mathrm{ZF}_{N}+\mathrm{DC}\left(\Delta_{1}^{2}\right)^{L(\mathbf{R})}$ sets of reals, consists of those sets of reals $A$ for which there are formulas $\varphi\left(v_{0}, v_{1}\right)$, $\psi\left(v_{0}, v_{1}\right)$ in $\Sigma_{1}^{2}, \Pi_{1}^{2}$ respectively, such that

$$
\mathrm{ZF}_{N}+\mathrm{DC} \vdash \varphi^{L(\mathbf{R})} \Leftrightarrow \psi^{L(\mathbf{R})}
$$

and

$$
A=\left\{\alpha \in \mathbf{R}: \varphi^{L(\mathbf{R})}\left(\alpha, x_{0}\right)\right\}
$$

for some parameter $x_{0} \in \mathbf{R}$, where as usual $\varphi^{M}$ denotes relativization to $M$. By the schema of provable (in $\mathrm{ZF}+\mathrm{DC})\left(\Delta_{1}^{2}\right)^{L(\mathrm{R})}$-DET we understand the infinite set of statements

$$
\left\{\operatorname{Pr}_{N}\left(\Delta_{1}^{2}\right)^{L(\mathbf{R})}-\mathrm{DET}\right\}_{N=1,2, \ldots},
$$

abbreviated by $\operatorname{Pr}\left(\Delta_{1}^{2}\right)^{L(R)}$-DET Schema.

We now have

THEOREM 2.3. (a) The following theories are equiconsistent:

(i) $\mathrm{ZFC}+L(\mathrm{R})-\mathrm{DET}$.

(ii) $\mathrm{ZFC}+\operatorname{Pr}\left(\Delta_{1}^{2}\right)^{L(\mathrm{R})}$-DET Schema.

(b) For each $N$,

$$
\mathrm{ZFC}+\operatorname{Pr}\left(\Delta_{1}^{2}\right)^{L(\mathbf{R})}-\mathrm{DET} \text { Schema } \vdash \operatorname{Con}\left(\mathrm{ZFC}+\operatorname{Pr}_{N}\left(\Delta_{1}^{2}\right)^{L(\mathbf{R})}-\mathrm{DET}\right) .
$$

1.3. There is an interesting phenomenon that occurs in the lowest levels of the preceding hierarchy, which can be seen easily by focusing on the "lightface" versions of some of the results we mentioned before. For instance we have:

$$
\begin{aligned}
& \text { TABLE } 2 \\
& \Pi_{1}^{1} \text {-DET } \quad \Leftrightarrow \quad 0^{\#} \text { exists } \\
& \hat{\imath} \\
& \left(\omega \cdot n-\Pi_{1}^{1}\right)-\mathrm{DET} \\
& \wedge \\
& \left(\omega^{2}+1-\Pi_{1}^{1}\right) \text {-DET } \quad \Leftrightarrow \quad \exists M(M \text { is an inner model with } \\
& \text { a measurable cardinal and there } \\
& \text { exists a class of indiscernibles } \\
& \text { for } \mathbf{M} \text { ) } \\
& \left(\omega^{2}+\omega \cdot n-\Pi_{1}^{1}\right)-\mathrm{DET} \\
& \wedge \\
& \Sigma_{1}^{0}\left(\Pi_{1}^{0}\right) \quad \Leftrightarrow \quad \exists M(M \text { is an inner model with } \\
& \text { arbitrarily large measurable } \\
& \text { cardinals and there exists a } \\
& \text { class of indiscernibles for } \mathbf{M} \text { ) }
\end{aligned}
$$


From these lightface results we see for example that

$$
\left(\omega^{2}+1-\Pi_{1}^{1}\right) \text {-DET (lightface!) }
$$

implies the existence of inner models with measurable cardinals, thus also the consistency of $\forall x \in \mathbf{R}$ ( $x^{\#}$ exists), i.e. the consistency of $\Pi_{1}^{1}$-DET (boldface!). So

$$
\left(\omega^{2}+1-\Pi_{1}^{1}\right) \text {-DET }>\Pi_{1}^{1} \text {-DET. }
$$

Similarly for the higher levels of the $\xi-\Pi_{1}^{1}$ hierarchy, and a little beyond. So we see that at the lowest levels (well within $\Delta_{2}^{1}$ ), $\Gamma$-DET for a boldface pointclass $\Gamma$ is strictly weaker than $\Gamma^{\prime}$-DET for some slightly bigger lightface pointclass $\Gamma^{\prime}$.

Could this persist higher up, for instance throughout the projective hierarchy so that, as H. Friedman asked,

$$
\mathrm{PD}\left(\equiv \text { Projective Determinancy } \equiv \bigcup_{n} \Sigma_{n}^{1} \text {-DET }\right)
$$

is equiconsistent to its lightface version $\bigcup_{n} \Sigma_{n}^{1}$-DET? The answer (in the negative) is given in the next theorem which shows surprisingly that the lightface hierarchy of determinacy hypotheses suddenly collapses at the level of $\Delta_{2}^{1}$-DET. (Compare this with the collapse of the boldface hierarchy at the level of $\left(\Delta_{1}^{2}\right)^{L(\mathbf{R})}$-DET).

THEOREM 4.1. The following theories are equiconsistent:

(i) $\mathrm{ZFC}+\Delta_{2}^{1}$-DET.

(ii) $\mathrm{ZFC}+\mathrm{OD}-\mathrm{DET}$.

Here OD is of course the class of all ordinal definable sets, so that OD-DET is the maximal lightface determinacy hypothesis.

Again except for a small further reduction to provable $\Delta_{2}^{1}$ determinacy the above result is best possible. More precisely we have the following:

THEOREM (PART OF 4.2). (a) The following theories are equiconsistent:

(i) ZFC + OD-DET.

(ii) $\mathrm{ZFC}+\operatorname{Pr} \Delta_{2}^{1}$-DET Schema.

(b) For each $N$,

$$
\mathrm{ZFC}+\operatorname{Pr} \Delta_{2}^{1} \text {-DET Schema } \vdash \operatorname{Con}\left(\mathrm{ZFC}+\operatorname{Pr}_{N} \Delta_{2}^{1} \text {-DET }\right) .
$$

We define $\operatorname{Pr}_{N} \Delta_{2}^{1}$ and $\operatorname{Pr} \Delta_{2}^{1}$-DET Schema as in $\$ 1.2$, i.e. $A \subseteq \mathbf{R}$ is $\operatorname{Pr}_{N} \Delta_{2}^{1}$ if there are formulas $\varphi(\alpha), \psi(\alpha)$ respectively in $\Sigma_{2}^{1}, \Pi_{2}^{1}$ such that $\mathrm{ZF}_{N}+\mathrm{DC} \vdash \varphi \Leftrightarrow \psi$ and $A=\{\alpha \in \mathbf{R}: \varphi(\alpha)\}$.

Theorem 4.1 and Martin's result (5) in Table 1 place an upper bound in terms of large cardinals to the probability of explicitly describing in ZFC an undetermined game. If we assume the consistency of the existence of a nontrivial iterable elementary embedding $j: V_{\lambda} \rightarrow V_{\lambda}$, then we have the consistency of OD-DET, so we cannot hope to establish a contradiction from the Definable Determinacy Hypothesis by actually exhibiting explicitly an undetermined game. Then one can concentrate on other possible approaches for attacking the consistency of this hypothesis. For instance, there is still a possibility that by pursuing further the already 
extensive structure theory of the continuum revealed by the Definable Determinacy Hypothesis, a contradiction will be discovered. In view of the way in which determinacy hypotheses are used in establishing these results (take for instance the Periodicity Theorems, [Mo1, Chapter 6]) one could not necessarily pinpoint an explicit undetermined game if such a contradiction arose. There could be for instance a definable family $\left\{A_{\alpha}\right\}_{\alpha \in \mathbf{R}}$ of games, so that one of them is undetermined, but we cannot explicitly specify which one.

1.4. We can actually establish the following result about the structure of the inner models $L[x]$ for $x \in \mathbf{R}$, which easily implies Theorem 4.1. It can be viewed as an analog of Theorem 2.1 for the models $L[x]$, instead of $L(\mathbf{R})$.

THEOREM 3.1. Assume ZF + DC. Then for all reals $x$,

$$
L[x] \vDash \Delta_{2}{ }^{2} \text { DET } \Rightarrow \text { OD-DET. }
$$

Equivalently, assuming $\mathrm{ZF}+\mathrm{DC}+\Delta_{2}^{1}$-DET, there is a real $y_{0}$ such that $L[x] \vDash$ OD-DET for all reals $x \geqslant_{T} y_{0}$, i.e. OD-DET holds in $L[x]$ for a cone of $x$ 's. ( Here $\leqslant_{T}$ is Turing reducibility).

Woodin has improved this result by showing that if $\Delta_{2}^{1}$-DET holds, there is a real $z_{0}$ such that for every additively closed ordinal $\lambda>\omega$ if $z_{0} \in L_{\lambda}[x], x$ a real, then $L_{\lambda}[x] \vDash$ OD-DET. We include a proof with his permission in $\S 3$ (see Theorem 3.2).

1.5. We return now to reconsider the phenomenon of the interplay between lightface and boldface determinacy hypotheses at low levels of $\Delta_{2}^{1}$-DET in the light of the preceding results. The next theorem shows that this phenomenon persists to the maximum extent allowed by the already established constraints.

THEOREM 4.2(a). The following theories are equiconsistent:

(i) ZFC + OD-DET.

(ii) $\mathrm{ZFC}+\operatorname{Pr} \Delta_{2}^{1}$-DET Schema.

(iii) $\mathrm{ZFC}+\operatorname{Pr} \Delta_{2}^{1}$..DET Schema.

Thus $\operatorname{Pr} \Delta_{2}^{1}$-DET Schema is essentially a maximal determinacy hypothesis for which the lightface and boldface forms are equiconsistent (it follows from the remarks in $\S 4$ that $\Delta_{2}^{1}$-DET is stronger that OD-DET).

Again every fixed level of provable $\Delta_{2}^{1}$ determinacy is weaker, in view of the following theorem.

THEOREM 4.2(b). For each $N$,

$$
\mathrm{ZFC}+\operatorname{Pr} \Delta_{2}^{1} \text {-DET Schema } \vdash \operatorname{Con}\left(\mathrm{ZFC}+\operatorname{Pr}_{N} \Delta_{2}^{1} \text {-DET }\right) .
$$

(Note the lightface hypothesis and boldface conclusion!)

1.6. We consider finally hierarchy theorems. Given pointclasses $\Gamma, \Gamma^{\prime}$ definable, say in $\mathrm{ZFC}$, when can we assert that $\Gamma^{\prime}$-DET $>\Gamma$-DET? We will study two main cases: (a) Lightface pointclasses contained in $\Delta_{2}^{1}$ vs. similar boldface ones, and (b) Boldface pointclasses contained in the projective sets. Various examples of hierarchy theorems under (b) were given in Table 1 , and we have seen some examples for (a) in $\$ \S 1.3$ and 1.5 . 
Actually we prove a general hierarchy theorem which covers both these cases and which gives in general best possible results. It is basically a generalization of the results of Martin in [Ma5]. We state our theorem and its implications precisely in §6.6, but we can now discuss its intuitive content in a somewhat less rigorous form.

Intuitively, our theorem asserts the following:

If $\Delta$ is a given "reasonable" self-dual pointclass contained strictly within the projective pointsets $\bigcup_{n} \Sigma_{n}^{1}$, such that $\Delta$ has the scale property (even in a slightly weaker sense than usual), then there is a single explicitly definable game $G$, such that if $G$ is determined, the theory " $\mathrm{ZFC}+\Delta$-DET" has a countable transitive model. The game $G$ will lie "just beyond" $\Delta$. In this way, we show that if $\Gamma$ is a lightface pointclass "just beyond" $\Delta$, then $\Gamma$-DET $>\Delta$-DET. "Just beyond" basically means here that $\Gamma$ has some reasonable closure properties, and contains a universal set for $\Delta$.

Let us see what this theorem says in the two cases (a), (b).

(a) Let $\Gamma_{0} \subseteq \Delta_{2}^{1}$ be a given boldface pointclass. To obtain a lightface pointclass $\Gamma_{1} \subseteq \Delta_{2}^{1}$ with $\Gamma_{1}$-DET $>\Gamma_{0}$-DET we need only go to a $\Gamma_{1}$ "just beyond" a self-dual "reasonable" pointclass $\Delta$ containing $\Gamma_{0}$, such that $\Delta$ has the scale property (in a weak sense), and this is the crucial condition. Such a $\Delta, \Gamma_{1}$ can always be found and will be also included in $\Delta_{2}^{1}, \Delta_{2}^{1}$ respectively: For instance, if $\Gamma_{0}=\Pi_{1}^{1}$ then we can take $\Delta=\bigcup_{n}\left(\omega \cdot n-\Pi_{1}^{1}\right)$ (by Steel [St2] this class has the weak scale property that we need), and so $\Gamma_{1}=\left(\omega^{2}+1\right)-\Pi_{1}^{1}$ is enough (and even less-see the Theorem in 8.1). Since (in ZF + DC) $\Pi_{1}^{1}$-DET $\Rightarrow\left(\omega \cdot n-\Pi_{1}^{1}\right)$-DET $\forall n<\omega$, this is a best possible estimate which demonstrates that our hierarchy theorem is in general best possible. In $\S 8$ we look at other concrete hierarchy theorems that can be obtained by applying the general theorem within the context of $\Delta_{2}^{1}$.

(b) Assume now that we start with a boldface pointclass $\Gamma_{0} \supseteq \Sigma_{2}^{1}$. Our general hierarchy theorem seems at first glance to be contrary to the preceding results mentioned in this introduction. For if $\Delta$ is a "reasonable" self-dual pointclass containing $\Gamma_{0}$, with the (weak) scale property, then there is a single ordinal definable game $G$ "just beyond" $\Delta$ such that the determinacy of $G$ implies the consistency of "ZFC $+\Delta$-DET" and thus of “ZFC $+\Gamma_{0}$-DET". In particular ZFC + OD-DET $\vdash$ $\operatorname{Con}\left(\mathrm{ZFC}+\Delta_{2}^{1}\right.$-DET), while it follows easily from our results in $\$ 4$ (see remarks following Theorem 4.2), that ZFC $+\Delta_{2}^{1}$-DET is stronger than ZFC + OD-DET.

If one analyzes the situation one gets the following: ZFC + OD-DET cannot prove (except via an inconsistency) that there is a class $\Delta$ containing $\Sigma_{2}^{1}$ and satisfying the hypotheses of our theorem, of which the crucial one is the (weak) scale property.

However, one can use various boldface determinacy hypotheses to obtain such classes $\Delta$ with the scale property. Let us consider a few examples.

(i) Assuming $\forall x \in \mathbf{R}$ ( $x^{\#}$ exists) one can show (see [St2, Mo1, 6E.15]) that $\Delta=\cup_{n} \supset\left(\omega \cdot n-\Pi_{1}^{1}\right)$, where $\supset$ is the game quantifier on $\omega$, has the (weak) scale property, as well as all the other simple properties required by our theory. Thus if $\Gamma_{1}$ is any lightface class "just beyond" $\Delta$, e.g. $\Gamma_{1}=\supset\left(\omega^{2}+1-\Pi_{1}^{1}\right)$, then

$$
\forall x \in \mathbf{R}\left(x^{\#} \text { exists }\right)+\Gamma_{1} \text {-DET }>\Delta \text {-DET, }
$$


in particular

$$
\forall x \in \mathbf{R}\left(x^{\#} \text { exists }\right)+\Gamma_{1} \text {-DET }>\Delta_{2}^{1} \text {-DET } .
$$

As an immediate corollary $\Delta_{3}^{1}$-DET $>\Delta_{2}^{1}$-DET (Martin [5]), see Table 1 .

By recent results of Kechris and Woodin [KeW],

$$
\mathrm{ZF}+\mathrm{DC}+\Delta_{2}^{1}-\mathrm{DET} \Rightarrow \bigcup_{n} \supset\left(\omega \cdot n-\Pi_{1}^{1}\right)-\mathrm{DET},
$$

so this hierarchy theorem is best possible here as well.

(ii) By generalizing (i) we have for instance that if for each $k \geqslant 0$ we let

$$
\mathbf{R}_{k}=\bigcup_{n}(\underbrace{\partial \supset \cdots \vartheta}_{k}\left(\omega \cdot n-\Pi_{1}^{1}\right)),
$$

then

$$
\mathbf{R}_{k} \text {-DET }+\Gamma_{k+1}-\mathrm{DET}>\Sigma_{k+2}^{1} \text {-DET, }
$$

where $\Gamma_{k+1}$ is "just beyond" $\mathbf{R}_{k+1}$, for example $\Gamma_{k+1}$ could be taken to be

$$
\underbrace{\partial \cdots \supset}_{k+1}\left(\omega^{2}+1-\Pi_{1}^{1}\right) \text {. }
$$

Note that $\mathbf{R}_{0}=\bigcup_{n}\left(\omega \cdot n-\Pi_{1}^{1}\right)$ and so by Martin-Harrington, in ZF + DC,

$$
\forall x \in \mathbf{R}\left(x^{\#} \text { exists }\right) \Leftrightarrow \mathbf{R}_{0} \text {-DET. }
$$

(For $k$ odd, it is shown in [KeW], that, in ZF + DC, $\mathbf{R}_{k}$-DET $\Leftrightarrow \Sigma_{k+1}^{1}$-DET, so we have for odd $k$ that $\Sigma_{k+1}^{1}$-DET $+\Gamma_{k+1}$-DET $>\Sigma_{k+2}^{1}$-DET). In particular, for $k \geqslant 0$

$$
\Delta_{k+3}^{1} \text {-DET }>\Sigma_{k+2}^{1} \text {-DET, }
$$

so these results are a sharpening of the hierarchy results for projective pointclasses in Table 1 . One can easily multiply now these examples ad infinitum.

The general moral is that within the projective hierarchy every boldface form of determinacy is consistencywise weaker than the conjunction of a much weaker boldface form together with a slightly higher lightface form.

Our main hierarchy theorem points out an interesting connection between the levels at which the scale property holds and the levels at which consistencywise stronger form of determinacy hypotheses are obtained. We have seen by examples that this is in general best possible, but it seems quite likely that it is always best possible, although a clear formulation of this conjecture and a proof of it are lacking at this moment.

1.7. We have divided the rest of this paper in two parts. The first $(\$ \S 2-5)$ contains the main equiconsistency results explained in $\$ \S 1.1-1.5$, while the second part $(\S \$ 6-8)$ is devoted to the main hierarchy theorem and its implications.

In general we follow standard set theoretic notation and terminology. We refer the reader to Moschovakis' book [Mo1] for concepts and results in descriptive set theory that we do not explicitly describe in this paper. 


\section{PART I: EQuiconsistency Results}

2. AD reduced to $\Delta_{1}^{2}$-DET in $L(\mathbf{R})$. We start with

THEOREM 2.1. ZF $+\mathrm{DC} \vdash\left(\Delta_{1}^{2}\right)^{L(\mathbf{R})}$-DET $\Rightarrow L(\mathbf{R})$-DET.

Assuming $\mathrm{ZF}+\mathrm{DC}+V=L(\mathbf{R})$, let $P(A) \Leftrightarrow A \subseteq \mathbf{R}$ and $A$ is not determined. Clearly $P$ is a projective predicate on power $(\mathbf{R})$. Thus Theorem 2.1 is an immediate consequence of the following basis theorem for $L(\mathbf{R})$.

TheOrem 2.2. Assume $\mathrm{ZF}+\mathrm{DC}+V=L(\mathbf{R})$. If $P(A)$ is a $\Sigma_{1}^{2}$ predicate on power(R), then $\exists A P(A) \Rightarrow \exists A \in \Delta_{1}^{2} P(A)$.

Proof. Work in ZF $+\mathrm{DC}+V=L(\mathbf{R})$. As every $\Sigma_{1}^{2}$ predicate $P(A)$ has the form $\exists B R(A, B)$, with $R$ projective, assume without loss of generality that $P(A)$ is already projective. Since $V=L(\mathbf{R})$, by standard results there is a definable (with no parameters) function $F:$ ORD $\times \mathbf{R} \stackrel{\text { onto }}{\rightarrow} V$. Thus we have that $\exists \xi \exists \alpha P(F(\xi, \alpha))$. Let $\lambda$ be at least such that

$$
L_{\lambda}(\mathbf{R}) \vDash \mathrm{ZF}_{N}+\mathrm{DC}+\exists \xi \exists \alpha P(F(\xi, \alpha)),
$$

where $N$ is a fixed large enough integer. A variant of the usual proof of the Skolem-Löwenheim theorem shows that $\lambda<\Theta$, where

$$
\Theta=\sup \{\xi: \text { There is a surjection } \pi: \mathbf{R} \stackrel{\text { onto }}{\rightarrow} \xi\} .
$$

Let $\alpha_{0} \in \mathbf{R}$ be such that $L_{\lambda}(\mathbf{R}) \vdash \exists \xi P\left(F\left(\xi, \alpha_{0}\right)\right)$. Finally let

$$
\alpha \in C \Leftrightarrow L_{\lambda}(\mathbf{R}) \vdash “ \exists \xi\left[P\left(F\left(\xi, \alpha_{0}\right)\right) \wedge \forall \xi^{\prime}<\xi \neg P\left(F\left(\xi, \alpha_{0}\right)\right) \wedge \alpha \in F\left(\xi, \alpha_{0}\right)\right] ” .
$$

Clearly $L_{\lambda}(\mathbf{R}) \vDash P(C)$, and since $P$ is projective we have by absoluteness that $P(C)$ holds as well. Now to see that $C \in \Delta_{1}^{2}$ note that if we abbreviate

$$
\sigma \Leftrightarrow \mathrm{ZF}_{N}+\mathrm{DC}+\exists \xi \exists \alpha P(F(\xi, \alpha)),
$$

then

$$
\begin{aligned}
\alpha \in C \Leftrightarrow \exists \lambda<\Theta & \left(L_{\lambda}(\mathbf{R}) \vDash \sigma \wedge \forall \lambda^{\prime}<\lambda L_{\lambda^{\prime}}(\mathbf{R}) \vDash \neg \sigma \wedge L_{\lambda}(\mathbf{R}) \vDash\right. \\
& \text { “ } \left.\exists \xi\left[P\left(F\left(\xi, \alpha_{0}\right)\right) \wedge \forall \xi^{\prime}<\xi \neg P\left(F\left(\xi, \alpha_{0}\right)\right) \wedge \alpha \in F\left(\xi, \alpha_{0}\right)\right] ”\right) .
\end{aligned}
$$

Since the structures $L_{\lambda}(\mathbf{R})$ for $\lambda<\Theta$ can be coded in a straightforward fashion by sets of reals, this shows that $C \in \Sigma_{1}^{2}$. A similar expression for $\sim C$ shows that $\sim C$ is also $\Sigma_{1}^{2}$, so $C \in \Delta_{1}^{2}$ and we are done.

We show now that the reduction from $L(\mathbf{R})$-DET to $\left(\Delta_{1}^{2}\right)^{L(\mathbf{R})}$-DET can be further improved to an optimal form.

THEOREM 2.3. (a) The following theories are equiconsistent:

(i) $\mathrm{ZFC}+L(\mathbf{R})-\mathrm{DET}$.

(ii) $\mathrm{ZFC}+\operatorname{Pr}\left(\Delta_{1}^{2}\right)^{L(\mathbf{R})}$-DET Schema.

(b) For each $N$,

$$
\mathrm{ZFC}+\operatorname{Pr}\left(\Delta_{1}^{2}\right)^{L(\mathbf{R})}-\mathrm{DET} \text { Schema } \vdash \operatorname{Con}\left(\mathrm{ZFC}+\operatorname{Pr}_{N}\left(\Delta_{1}^{2}\right)^{L(\mathbf{R})}-\mathrm{DET}\right) .
$$


Proof. (a) Assume ZFC $+L(\mathbf{R})$-DET $\vdash 0=1$. Then also $\mathrm{ZF}+\mathrm{DC}+\mathrm{AD}+V$ $=L(\mathbf{R}) \vdash 0=1$. We shall deduce that $\mathrm{ZFC}+\operatorname{Pr}\left(\Delta_{1}^{2}\right)^{L(\mathbf{R})}$-DET Schema $\vdash 0=1$.

Let $N$ be large enough such that $\mathrm{ZF}_{N}+\mathrm{DC}+\mathrm{AD}+V=L(\mathbf{R}) \vdash 0=1$ and that various things that are needed below are provable in $\mathrm{ZF}_{N}+\mathrm{DC}+V=L(\mathbf{R})$. Then $\mathrm{ZF}_{N}+\mathrm{DC}+V=L(\mathbf{R}) \vdash \neg \mathrm{AD}$. Let $F(\xi, \alpha)$ be the canonical definable (with no parameters) function mapping $\mathrm{ORD} \times \mathbf{R}$ onto $V$, assuming $\mathrm{ZF}_{N}+\mathrm{DC}+V=L(\mathbf{R})$.

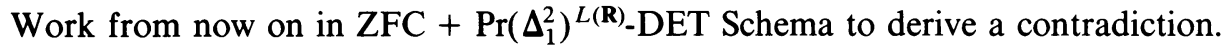
Let $\lambda_{N}$ be the least ordinal $\lambda$ such that $L_{\lambda}(\mathbf{R}) \vDash " Z F_{N}+\mathrm{DC}+V=L(\mathbf{R})$ ". Then since also

$$
L_{\lambda_{N}}(\mathbf{R}) \vDash “ \neg \mathrm{AD}+F: \text { ORD } \times \mathbf{R} \stackrel{\text { onto }}{\rightarrow} V \text { ", }
$$

we have

$$
\begin{aligned}
L_{\lambda_{N}}(\mathbf{R}) \vDash \text { " } & \text { For some }\left(\xi_{0}, \beta_{0}\right) \in \text { ORD } \times \mathbf{R}, \\
& F\left(\xi_{0}, \beta_{0}\right) \text { is an undetermined game". }
\end{aligned}
$$

Put

$$
\begin{aligned}
(\alpha, \beta) \in D \Leftrightarrow L_{\lambda_{N}}(\mathbf{R}) \vDash & \text { “ } \exists \xi(F(\xi, \beta) \text { is not } \\
& \text { determined } \wedge \forall \xi^{\prime}<\xi F\left(\xi^{\prime}, \beta\right) \text { is } \\
& \text { determined } \wedge \alpha \in F(\xi, \beta)) " .
\end{aligned}
$$

Pick $\beta_{0} \in \mathbf{R}$ such that $L_{\lambda_{N}}(\mathbf{R}) \vDash \exists \xi\left(F\left(\xi, \beta_{0}\right)\right.$ is undetermined). Then if $\alpha \in A \Leftrightarrow$ $\left(\alpha, \beta_{0}\right) \in D$, clearly $A$ is in $\operatorname{Pr}_{M}\left(\Delta_{1}^{2}\right)^{L(\mathbf{R})}$ for some large enough $M$, and $A$ is not determined by absoluteness of this statement between $L_{\lambda_{N}}(\mathbf{R})$ and the universe. This contradicts $\operatorname{Pr}\left(\Delta_{1}^{2}\right)^{L(\mathbf{R})}$-DET Schema.

(b) Fix $N$. We will show that

$$
\mathrm{ZFC}+\operatorname{Pr}\left(\Delta_{1}^{2}\right)^{L(\mathbf{R})}-\mathrm{DET} \text { Schema } \vdash \operatorname{Con}\left(\mathrm{ZFC}+\operatorname{Pr}_{N}\left(\Delta_{1}^{2}\right)^{L(\mathbf{R})}-\mathrm{DET}\right) .
$$

As in the preceding proofs we can find, working in $\mathrm{ZFC}+\operatorname{Pr}\left(\Delta_{1}^{2}\right)^{L(\mathbf{R})}$-DET Schema, a $\lambda<\Theta^{L(R)}$ such that

$$
L_{\lambda}(\mathbf{R}) \vdash " \mathrm{ZF}_{N}+\mathrm{DC}+V=L(\mathbf{R})+\mathrm{AD} " .
$$

Let $M=\operatorname{HOD}^{L_{\lambda}(\mathbf{R})}, M^{\prime}=\left\{x \in M: \operatorname{rank}^{M}(x)<\omega_{1}^{L_{\lambda}(\mathbf{R})}\right\}$. Since Solovay's proof (see [Ke2]) that $\mathrm{ZF}+\mathrm{DC}+\mathrm{AD} \vdash$ " $\omega_{1}$ is measurable" shows also that $\mathrm{ZF}+\mathrm{DC}+\mathrm{AD}$ $\vdash$ "the $\omega_{1}$ of the universe is measurable in HOD", it follows that $\omega_{1}^{L_{\lambda}(R)}$ is measurable in $M$, so $M^{\prime} \vDash \mathrm{ZFC}$ (we are assuming here that $N$ has been chosen large enough). So it is enough to check that $M \vDash \operatorname{Pr}_{N}\left(\Delta_{1}^{2}\right)^{L(\mathbf{R})}$-DET. So let $\varphi, \psi$ be respectively $\Sigma_{1}^{2}, \Pi_{1}^{2}$ formulas such that $M \vDash " Z \mathrm{ZF}_{N}+\mathrm{DC} \vdash \varphi^{L(\mathbf{R})} \Leftrightarrow \psi^{L(\mathbf{R})}$ ", and let $\beta_{0} \in M$ be a fixed real. We want to prove that

$$
M \vDash "\left\{\alpha: \varphi::^{L(\mathbf{R})}\left(\alpha, \beta_{0}\right)\right\} \text { is determined". }
$$

Since $M$ is a standard model (therefore in particular an $\omega$-model), we have that $\mathrm{ZF}_{N}+\mathrm{DC} \vdash \varphi^{L(\mathbf{R})} \Leftrightarrow \psi^{L(\mathbf{R})}$ and so $L_{\lambda}(\mathbf{R}) \vDash \varphi^{L(\mathbf{R})} \Leftrightarrow \psi^{L(\mathbf{R})}$. So $L_{\lambda}(\mathbf{R}) \vDash$ “ $\left\{\alpha: \varphi^{L(\mathbf{R})}\left(\alpha, \beta_{0}\right)\right\}=\left\{\alpha: \psi^{L(\mathbf{R})}\left(\alpha, \beta_{0}\right)\right\}$ is a $\Delta_{1}^{2}\left(\beta_{0}\right)^{L(\mathbf{R})}$ set". As also $L_{\lambda}(\mathbf{R}) \vDash$ " $\left\{\alpha: \varphi^{L(\mathbf{R})}\left(\alpha, \beta_{0}\right)\right\}$ is determined", assume without loss of generality that

$$
L_{\lambda}(\mathbf{R}) \vDash \text { "I has a winning strategy for }\left\{\alpha: \varphi^{L(\mathbf{R})}\left(\alpha, \beta_{0}\right)\right\} \text { ". }
$$


By the work of Martin, Moschovakis and Steel (see [MMS, MSt]), we have that

$$
\begin{aligned}
\mathrm{ZF}+\mathrm{AD}+\mathrm{DC}+V=L(\mathbf{R}) \vdash & \text { "Every nonempty } \Sigma_{1}^{2}(\beta) \text { set } \\
& \text { of reals } A \text { contains a } \Delta_{1}^{2}(\beta) \text { member". }
\end{aligned}
$$

So (again assuming $N$ is large enough) we have that

$$
\begin{aligned}
L_{\lambda}(\mathbf{R}) \vDash & \text { "I has a winning strategy } \sigma \text { for } \\
& \left\{\alpha: \varphi^{L(\mathbf{R})}\left(\alpha, \beta_{0}\right)\right\} \text { which lies in HOD". }
\end{aligned}
$$

It is finally enough to check that

$$
M \vDash \text { " } \sigma \text { is a winning strategy for the player I for }\left\{\alpha, \varphi^{L(\mathbf{R})}\left(\alpha, \beta_{0}\right)\right\} " \text {. }
$$

This however follows immediately from the following corollary of the work in [MSt], noticed by Woodin.

Lemma. Assume $\mathrm{ZF}+\mathrm{AD}+\mathrm{DC}+V=L(\mathbf{R})$. For every $\Sigma_{1}^{2}$ formula $\varphi$ and parameter $\alpha \in \mathrm{HOD}$, we have $\varphi(\alpha) \Rightarrow \mathrm{HOD} \vDash \varphi(\alpha)$.

Proof of The Lemma. Work in ZF $+\mathrm{AD}+\mathrm{DC}+V=L(\mathbf{R})$. By [MSt] if $P(A)$ is a $\Sigma_{1}^{2}(\alpha)$ predicate on power $(\mathbf{R})$, then

$$
\exists A P(A) \Rightarrow \exists A \in \Delta_{1}^{2}(\alpha) P(A) .
$$

So if $\varphi(\alpha) \Leftrightarrow \exists A P(A, \alpha)$, where $P$ is analytical, then $\varphi(\alpha) \Rightarrow \exists A \in \Delta_{1}^{2}(\alpha) P(A, \alpha)$. It is easy now to verify using the fact that every nonempty $\Delta_{1}^{2}(\beta)$ set of reals contains a $\Delta_{1}^{2}(\beta)$ member that for $\alpha \in \mathrm{HOD}, A \in \Delta_{1}^{2}(\alpha)$ we have

$$
P(A, \alpha) \Rightarrow \mathrm{HOD} \vDash P(A \cap \mathrm{HOD}, \alpha),
$$

which completes our proof of the lemma and the theorem.

3. OD-DET reduced to $\Delta_{2}^{1}$-DET in $L[x], x \in \mathbf{R}$. The following can be thought of as a lightface analog for $L[x], x \in \mathbf{R}$, of Theorem 2.1.

THEOREM 3.1. Assume ZF + DC. Then for all reals $x$,

$$
L[x] \vDash \Delta_{2}^{1} \text {-DET } \Rightarrow \text { OD-DET. }
$$

Equivalently, assuming $\mathrm{ZF}+\mathrm{DC}+\Delta_{2}^{1}$-DET, there is a real $y_{0}$ such that $L[x] \vDash$ OD-DET for all reals $x \geqslant_{T} y_{0}$.

Proof. To see that these two statements are equivalent just notice that $\Delta_{2}^{1}$-DET is a $\Sigma_{3}^{1}$ sentence, thus it relativizes downward to all $L[x]$ 's, $x \geqslant_{T} y_{0}$, for some fixed real $y_{0}$.

We now prove the second version. Work below in $\mathrm{ZF}+\mathrm{DC}+\Delta_{2}^{1}$-DET. By Martin [Ma5] we also have $\Sigma_{2}^{1}$-DET (for an alternative proof see $\S 5$ below). Pick $N$ large enough such that all the results from $\mathrm{ZFC}$ that we need below are provable in $\mathrm{ZF}_{N}+\mathrm{AC}$. If there is no $y_{0}$ such that for all $x \geqslant_{T} y_{0}$ we have $L[x] \vDash \mathrm{OD}$-DET, then

$$
\forall z \exists y \geqslant_{T} z(L[y] \vDash \neg \mathrm{OD}-\mathrm{DET}) .
$$

So by Skolem-Löwenheim

$$
\forall z \exists y \geqslant_{T} z \exists \vartheta<\omega_{1}\left(L_{\vartheta}[y] \vDash \mathrm{ZF}_{N}+\mathrm{AC}+\neg \mathrm{OD}-\mathrm{DET}\right) .
$$


But $\left\{y: \exists \vartheta<\omega_{1}\left(L_{\vartheta}[y] \vDash \mathrm{ZF}_{N}+\mathrm{AC}+\neg \mathrm{OD}-\mathrm{DET}\right)\right\}$ is a $\Sigma_{2}^{1}$ Turing invariant set of reals, thus by $\Sigma_{2}^{1}$-DET,

$$
\exists z_{0} \forall y \geqslant_{T} z_{0} \exists \vartheta<\omega_{1}\left(L_{\vartheta}[y] \vDash \mathrm{ZF}_{N}+\mathrm{AC}+\neg \mathrm{OD}-\mathrm{DET}\right) .
$$

Put

$$
\vartheta(y)=\left\{\begin{array}{l}
\text { least } \vartheta \text { such that } L_{\vartheta}[y] \vDash " Z \mathrm{ZF}_{N}+\mathrm{AC}+\neg \mathrm{OD}-\mathrm{DET} " \text { if such exists, } \\
\text { undefined otherwise. }
\end{array}\right.
$$

Thus $\exists z_{0} \forall y \geqslant_{T} z_{0}(\vartheta(y)$ is defined).

LEMMA. $\forall w \exists v \geqslant_{T} w \forall y \geqslant_{T} v(\vartheta(y) \geqslant \vartheta(v))$.

Proof. Otherwise there is a $w_{0}$ such that for all $v \geqslant_{T} w_{0}$ there is $y \geqslant_{T} v$ with $\vartheta(y)<\vartheta(v)$. This clearly leads to an infinite descending chain of ordinals.

Consider now the formula $<_{\text {oD }}$ defining the canonical wellordering of OD sets in $\mathrm{ZF}_{N}+\mathrm{AC}$. Let $\varphi(\alpha)$ be the formula defining the $<_{\mathrm{OD}^{-}}$-least undetermined OD sets of reals, if such exists, and $\varnothing$ otherwise, i.e.

$$
\begin{aligned}
\varphi(\alpha) \Leftrightarrow \exists A \in \mathrm{OD}\{A \subseteq \mathbf{R} \wedge A \text { is not determined } \\
\left.\qquad \forall B<{ }_{\mathrm{OD}} A(B \subseteq \mathbf{R} \Rightarrow B \text { is determined }) \wedge \alpha \in A\right\} .
\end{aligned}
$$

Define then the following game $G$ :

$$
\begin{array}{ll}
\underset{\alpha, x_{0}}{\text { I }} \quad \text { II }_{x_{1}, \beta} & \text { Let } x=\left\langle x_{0}, x_{1}\right\rangle ; \text { then I wins iff } \\
& \vartheta(\langle\alpha, x, \beta\rangle) \text { is defined and } \\
& L_{\vartheta(\langle\alpha, x, \beta\rangle)}[\langle\alpha, x, \beta\rangle] \vDash \varphi(\langle\alpha, \beta\rangle),
\end{array}
$$

where $\langle\alpha, \beta\rangle=(\alpha(0), \beta(0), \ldots)$ and similarly for $\langle\alpha, \beta, \gamma\rangle$. This is a $\Sigma_{2}^{1}$ game, so it is determined. Say I has a winning strategy $\sigma$ (the argument is similar for II).

Let $v_{0} \geqslant_{T} \sigma$ be such that for $x \geqslant_{T} v_{0}, \vartheta(x)$ is defined and $\vartheta(x) \geqslant \vartheta\left(v_{0}\right)$. We claim that $L_{\vartheta\left(v_{0}\right)}\left[v_{0}\right] \vDash "\{x: \varphi(\alpha)\}$ is determined", which is a contradiction, since $L_{\vartheta\left(v_{0}\right)}\left[v_{0}\right] \vDash \neg$ OD-DET.

Indeed, working in $L_{\vartheta\left(v_{0}\right)}\left[v_{0}\right]$, if II plays $\beta$, let I play the $\alpha$ such that (for the appropriate $\left.x_{0}\right)\left(\alpha, x_{0}\right)=\sigma\left(v_{0}, \beta\right)$, i.e. $\left(\alpha, x_{0}\right)$ is what I plays in $G$ following $\sigma$ when II plays $v_{0}, \beta$. Then

$$
L_{\vartheta\left(\left\langle\alpha,\left\langle x_{0}, v_{0}\right\rangle, \beta\right\rangle\right)}\left[\left\langle\alpha,\left\langle x_{0}, v_{0}\right\rangle, \beta\right\rangle\right] \vDash \varphi(\langle\alpha, \beta\rangle),
$$

so it is enough to show that

$$
L_{\vartheta\left(v_{0}\right)}\left[v_{0}\right]=L_{\vartheta\left(\left\langle\alpha,\left\langle x_{0}, v_{0}\right\rangle, \beta\right\rangle\right)}\left[\left\langle\alpha,\left\langle x_{0}, v_{0}\right\rangle, \beta\right\rangle\right]
$$

to demonstrate that this is a winning strategy for $I$ in $\{\alpha: \varphi(\alpha)\}$, within $L_{\vartheta\left(v_{0}\right)}\left[v_{0}\right]$. Since

$$
L_{\vartheta\left(v_{0}\right)}\left[\left\langle\alpha,\left\langle x_{0}, v_{0}\right\rangle, \beta\right\rangle\right]=L_{\vartheta\left(v_{0}\right)}\left[v_{0}\right] \vDash \mathrm{ZF}_{N}+\mathrm{AC}+\neg \mathrm{OD}-\mathrm{DET},
$$


we clearly have that

$$
L_{\vartheta\left(\left\langle\alpha,\left\langle x_{0}, v_{0}\right\rangle, \beta\right\rangle\right)}\left[\left\langle\alpha,\left\langle x_{0}, v_{0}\right\rangle, \beta\right\rangle\right] \subseteq L_{\vartheta\left(v_{0}\right)}\left[v_{0}\right] .
$$

But also $v_{0} \leqslant{ }_{T}\left\langle\alpha,\left\langle x_{0}, v_{0}\right\rangle, \beta\right\rangle$, thus $\vartheta\left(v_{0}\right) \leqslant \vartheta\left(\left\langle\alpha,\left\langle x, v_{0}\right\rangle, \beta\right\rangle\right)$ by the choice of $v_{0}$. Therefore

$$
L_{\vartheta\left(v_{0}\right)}\left[v_{0}\right] \subseteq L_{\vartheta\left(\left\langle\alpha,\left\langle x_{0}, v_{0}\right\rangle, \beta\right\rangle\right)}\left[\left\langle\alpha,\left\langle x_{0}, v_{0}\right\rangle, \beta\right\rangle\right]
$$

and we are done.

The following improvement of Theorem 3.1 is due to Woodin.

Theorem 3.2 (Woodin). Assume ZF $+\mathrm{DC}+\Delta_{2}^{1}$-DET. Then there is a real $z_{0}$ such that for every additively closed ordinal $\lambda>\omega$, if $z_{0} \in L_{\lambda}[x], x$ a real, then $L_{\lambda}[x] \vDash$ OD-DET.

Since $L_{\lambda}[x]$ may satisfy very little of ZFC, we think here of " $L_{\lambda}[x] \vDash$ OD-DET" as an abbreviation of the (external in general to $L_{\lambda}[x]$ ) statement: For each ordinal $\eta<\lambda$ and each formula $\varphi$ of the language of set theory, $L_{\lambda}[x] \vDash$ "There is a winning strategy for player I or II in the game $\{\alpha \in \mathbf{R}: \varphi(\alpha, \xi)\}$ ". (Note that in $L_{\lambda}[x]$, $\{\alpha \in \mathbf{R}: \varphi(\alpha, \xi)\}$ may not be even a set, but the notion of a winning strategy makes perfectly good sense.)

With these explanations we give now the

Proof of The Theorem. Assume towards a contradiction that for all reals $z$, there is an additively closed ordinal $\lambda>\omega$ such that for some real $x$ with $z \in L_{\lambda}[x]$, $L_{\lambda}[x] \not$ OD-DET. Note the following simple fact now: If $\lambda$ is additively closed and $\lambda>\omega$, then for reals $y, x$ :

$$
y \in L_{\lambda}[x] \Rightarrow L_{\lambda}[y] \subseteq L_{\lambda}(x) .
$$

Thus we conclude that for each real $z$ there is an additively closed ordinal $\lambda>\omega$ and a real $y$ with $z \leqslant_{T} y$ such that $L_{\lambda}[x] \not \equiv$ OD-DET. (Take an $x$ as before and let $y$ $=\langle z, x\rangle$.) By Skolem-Löwenheim we have now that

$$
\forall z \exists y \geqslant_{T} z \exists \lambda\left(\lambda<\omega_{1} \wedge \lambda>\omega \wedge \lambda \text { is additively closed } \wedge L_{\lambda}[y] \not \equiv \text { OD-DET }\right) .
$$

Thus using $\Sigma_{2}^{1}$-DET we have that there is some $y_{0}$ such that for all $y \geqslant_{T} y_{0}$ there is an additively closed countable ordinal $\lambda>\omega$ with $L_{\lambda}[y] \not \neq$ OD-DET.

Define

$$
\lambda(y)=\left\{\begin{array}{l}
\text { the least countable additively closed } \lambda>\omega, \\
\text { with } L_{\lambda}[y] \not \neq \text { OD-DET } \\
\text { undefined otherwise. }
\end{array}\right.
$$

Thus $y \geqslant y_{0} \Rightarrow \lambda(y)$ is defined. Also as in the proof of Theorem 3.1, $\forall w \exists v \geqslant_{T} w \forall y \geqslant_{T} v(\lambda(y) \geqslant \lambda(v))$. Finally for each $y \geqslant_{T} y_{0}$ let $\left(\varphi_{y}, \eta_{y}\right)$ be lexicographically least such that $L_{\lambda(y)}[y] \vDash$ " $\left\{\alpha \in \mathbf{R}: \varphi_{y}\left(\alpha, \eta_{y}\right)\right\}$ is not determined". Note that $\varphi_{y}, \eta_{y}$ depend only on $L_{\lambda[y]}[y]$, i.e. if $L_{\lambda[x]}[x]=L_{\lambda[y]}[y]$, then $\left(\varphi_{y}, \eta_{y}\right)=$ $\left(\varphi_{x}, \eta_{x}\right)$. 
Consider now as in the proof of Theorem 3.1 the game $G$ :

$$
\begin{array}{ccl}
\text { I } & \text { II } & \text { Let } x=\left\langle x_{0}, x_{1}\right\rangle ; \text { then I wins iff } \\
\alpha, x_{0} & x_{1}, \beta & \lambda(\langle\alpha, x, \beta\rangle) \text { is defined and } \\
& & L_{\lambda(\langle\alpha, x, \beta\rangle)}[\langle\alpha, x, \beta\rangle] \vDash \varphi_{\langle\alpha, x, \beta\rangle}\left(\langle\alpha, \beta\rangle, \eta_{\langle\alpha, x, \beta\rangle}\right) .
\end{array}
$$

We can now repeat the rest of the argument in this proof, using again the fact that if $\lambda$ is additively closed and $y \in L_{\lambda}[x]$, then $L_{\lambda}[y] \subseteq L_{\lambda}[x]$.

4. Lightface determinacy. As an immediate corollary of Theorem 3.1 we have the following

THEOREM 4.1. The following theories are equiconsistent:

(i) ZFC + OD-DET.

(ii) $\mathrm{ZFC}+\Delta_{2}^{1}$-DET.

Our next result improves this to an optimal form and establishes the equiconsistency of lightface and boldface forms of determinacy at this level.

THEOREM 4.2. (a) The following theories are equiconsistent:

(i) ZFC + OD-DET.

(ii) $\mathrm{ZFC}+\operatorname{Pr} \Delta_{2}^{1}$-DET Schema.

(iii) $\mathrm{ZFC}+\operatorname{Pr} \Delta_{2}^{1}$-DET Schema.

(b) For each $N$,

$$
\mathrm{ZFC}+\operatorname{Pr} \Delta_{2}^{1} \text {-DET Schema } \vdash \operatorname{Con}\left(\mathrm{ZFC}+\operatorname{Pr}_{N} \Delta_{2}^{1} \text {-DET }\right) .
$$

Proof. We show first that (i), (ii) are equiconsistent. Assume ZFC + OD-DET $\vdash$ $0=1$. We shall deduce that $\mathrm{ZFC}+\operatorname{Pr} \Delta_{2}^{1}$-DET Schema $\vdash 0=1$. Let $N$ be large enough such that $\mathrm{ZF}_{N}+\mathrm{AC}+\mathrm{OD}-\mathrm{DET} \vdash 0=1$, and various facts about $\mathrm{ZFC}$ that are needed below are provable in $\mathrm{ZF}_{N}+\mathrm{AC}$. Then $\mathrm{ZF}_{N}+\mathrm{AC} \vdash \neg \mathrm{OD}-\mathrm{DET}$. Let $<_{\text {oD }}$ be the formula defining the canonical wellordering of $\mathrm{OD}$ in $\mathrm{ZF}_{N}+\mathrm{AC}$. Let $\varphi(\alpha)$ be the formula defining the $<_{{ }_{O D}}$-least undetermined OD set of reals, if such exists, and $\varnothing$ otherwise, as in the proof of Theorem 3.1.

Work now in ZFC $+\operatorname{Pr} \Delta_{2}^{1}$-DET Schema to prove a contradiction. For $x \in \mathbf{R}$, let $\vartheta_{N}(x)$ be the least ordinal $\vartheta$ such that $L_{\vartheta}[x] \vDash \mathrm{ZF}_{N}+\mathrm{AC}$. Consider then the following game $G$ :

$$
\begin{array}{lll}
\underset{\alpha, x_{0}}{\text { I }} & \text { II } & \text { Let } x=\left\langle x_{0}, x_{1}\right\rangle ; \text { I wins iff } \\
& & L_{\vartheta_{N}(\langle\alpha, x, \beta\rangle)}[\langle\alpha, x, \beta\rangle] \vDash \varphi(\langle\alpha, \beta\rangle) .
\end{array}
$$

This game is clearly in $\operatorname{Pr}_{M} \Delta_{2}^{1}$ for some large enough $M$, so it is determined. Say I has a winning strategy $\sigma$ (the argument is similar for II). We claim that

$$
L_{\vartheta_{N}(\sigma)}[\sigma] \vDash \text { "I has a winning strategy in }\{\alpha: \varphi(\alpha)\} \text { ", }
$$

which is a contradiction as $L_{\vartheta_{N}(\sigma)}[\sigma] \vDash \mathrm{ZF}_{N}+\mathrm{AC}$, therefore $L_{\vartheta_{N}(\sigma)}[\sigma] \vDash \neg \mathrm{OD}-\mathrm{DET}$, therefore $L_{\vartheta_{N}(\sigma)}[\sigma] \vDash "\{\alpha: \varphi(\alpha)\}$ is not determined". 
Indeed, if II plays any $\beta \in \mathbf{R}$ in $L_{\vartheta_{N}(\sigma)}[\sigma]$, then I answers by playing the $\alpha$ such that $\left(\alpha, x_{0}\right)=\sigma(\sigma, \beta)$, i.e. $\alpha, x_{0}$ is what I plays in the game $G$ when he follows $\sigma$ and II plays $\left(x_{1}, \beta\right) \equiv(\sigma, \beta)$. We claim that this is a winning strategy for I in $L_{\vartheta_{N}(\sigma)}[\sigma]$ for $\{\alpha: \varphi(\alpha)\}$. Clearly by the rules of $G$,

$$
L_{\vartheta_{N}\left(\left\langle\alpha,\left\langle x_{0}, \sigma\right\rangle, \beta\right\rangle\right)}\left[\left\langle\alpha,\left\langle x_{0}, \sigma\right\rangle, \beta\right\rangle\right] \vDash \varphi(\langle\alpha, \beta\rangle),
$$

so it is enough to check that

$$
L_{\vartheta_{N}\left(\left\langle\alpha,\left\langle x_{0}, \sigma\right\rangle, \beta\right\rangle\right)}\left[\left\langle\alpha,\left\langle x_{0}, \sigma\right\rangle, \beta\right\rangle\right]=L_{\vartheta_{N}(\sigma)}[\sigma] .
$$

But this is immediate from the fact that the theory $\mathrm{ZF}_{N}$ relativizes to inner models of the form $L[y]$ for $y$ a real (see for example Gostanian [G]).

To prove that (ii) and (iii) are equiconsistent and (b) it is enough to establish the latter. So fix large enough $N$. We shall prove that

$$
\mathrm{ZFC}+\operatorname{Pr} \Delta_{2}^{1} \text {-DET Schema } \vdash \operatorname{Con}\left(\mathrm{ZFC}+\operatorname{Pr}_{N} \Delta_{2}^{1} \text {-DET }\right)
$$

The idea is similar to that in the proof of Theorem 2.3(b).

As in the preceding argument we can find an $x \in \mathbf{R}$ and $\vartheta<\omega_{1}$ such that

$$
L_{\vartheta}[x] \vDash \mathrm{ZF}_{N}+\mathrm{AC}+\mathrm{OD}-\mathrm{DET} \text {. }
$$

Let $M=\operatorname{HOD}^{L_{\vartheta}[x]}, M^{1}=\left\{z \in M: \operatorname{rank}^{M}(z)<\omega_{1}^{L_{\vartheta}[x]}\right\}$. Since $L_{\vartheta}[x] \vDash$ OD-DET we can see as in the proof of 2.2 that $\omega_{1}^{L_{\vartheta}[x]}$ is measurable in $M$, so $M^{1} \vDash$ ZFC. So it is enough to check that $M \vDash \operatorname{Pr}_{N} \Delta_{2}^{1}$-DET. So let $\chi, \psi$ be respectively $\Sigma_{2}^{1}, \Pi_{2}^{1}$ formulas such that $M \vDash$ " $\mathrm{ZF}_{N}+\mathrm{DC} \vdash \chi \Leftrightarrow \psi$ ". Let $\beta_{0} \in M$. We want to prove that $M \vDash "\left\{\alpha: \chi\left(\alpha, \beta_{0}\right)\right\}$ is determined". Since $M$ is an $\omega$-model we clearly have that $\mathrm{ZF}_{N}+\mathrm{DC} \vdash \chi \Leftrightarrow \psi$, thus we also have that $L_{\vartheta}(x) \vDash \chi \Leftrightarrow \psi$. So $L_{\vartheta}[x] \vDash$ " $\{\alpha$ : $\left.\chi\left(\alpha, \beta_{0}\right)\right\}$ is a $\Delta_{2}^{1}\left(\beta_{0}\right)$ set". Since $\beta_{0} \in \operatorname{HOD}^{L_{\vartheta}[x]}$, the proof of the 3rd Periodicity Theorem of Moschovakis [Mo1] applies (since $L_{\vartheta}[x] \vDash$ OD-DET) to show that $L_{\vartheta}[x] \vDash$ "there is a $\Delta_{3}^{1}\left(\beta_{0}\right)$ winning strategy in $\left\{\alpha: \chi\left(\alpha, \beta_{0}\right)\right\}$ ". So there is a real $\sigma \in M$ such that $L_{\vartheta}[x] \vDash$ " $\sigma$ is a winning strategy for I or II in $\left\{\alpha: \chi\left(\alpha, \beta_{0}\right)\right\}$ ". This is a $\Pi_{3}^{1}$ statement about $\sigma, \beta_{0} \in M$ so it relativizes down to $M$, i.e. $M \vDash$ " $\{\alpha$ : $\left.\chi\left(\alpha, \beta_{0}\right)\right\}$ is determined" and our proof is complete.

REMARK. Let $\operatorname{Pr} \Delta_{2}^{1}$-DET be the single sentence asserting the determinacy of all provable in ZFC $\Delta_{2}^{1}$ sets of reals. Similarly for $\operatorname{Pr} \Delta_{2}^{1}$-DET. By Theorem 4.2, $\mathrm{ZFC}+\operatorname{Pr} \Delta_{2}^{1}$-DET is equiconsistent with $\mathrm{ZFC}+\operatorname{Pr} \Delta_{2}^{1}$-DET Schema. As opposed to that, $\mathrm{ZFC}+\operatorname{Pr} \Delta_{2}^{1}$-DET is consistencywise stronger than $\mathrm{ZFC}+\operatorname{Pr} \Delta_{2}^{1}$-DET Schema. This is because from the immediately preceding proof we see that if $N_{0}$ is large enough, then for each $N$ :

$$
\begin{aligned}
& \mathrm{ZF}_{N_{0}}+\mathrm{AC}+\operatorname{Pr} \Delta_{2}^{1} \text {-DET Schema }+\forall x \in \mathbf{R} \exists M \\
& (M \text { is a standard (set) model of ZFC containing } x) \\
& \vdash \operatorname{Con}\left(\mathrm{ZFC}+\operatorname{Pr}_{N} \Delta_{2}^{1} \text {-DET }\right) .
\end{aligned}
$$

Then

$$
\begin{aligned}
& \operatorname{Con}\left(\mathrm{ZF}_{N_{0}}+\mathrm{AC}+\operatorname{Pr} \Delta_{2}^{1}-\mathrm{DET} \text { Schema }+\forall x \in \mathbf{R} \exists M\right. \\
& (M \text { is a standard model of } \mathrm{ZFC} \text { containing } x)) \\
& \Rightarrow \operatorname{Con}\left(\mathrm{ZFC}+\operatorname{Pr} \Delta_{2}^{1} \text {-DET Schema }\right) .
\end{aligned}
$$


But also

$\mathrm{ZFC}+\operatorname{Pr} \Delta_{2}^{1}-\mathrm{DET} \vdash \operatorname{Con}\left(\mathrm{ZF}_{N_{0}}+\mathrm{AC}+\operatorname{Pr} \Delta_{2}^{1}\right.$-DET Schema $+\forall x \in \mathbf{R} \exists M$

( $M$ is a standard model of ZFC containing $x)$ ).

This is because

(1) The statement " $\forall x \in \mathbf{R} \exists M$ ( $M$ is a standard model of ZFC containing $x$ )" follows from " $\forall x \in \mathbf{R}$ ( $x$ \# exists)" and hence from $\Pi_{1}^{1}$-DET, and so a fortiori from $\operatorname{Pr} \Delta_{2}^{1}$-DET.

(2) By the standard reflection principle, “ZFC $+\operatorname{Pr} \Delta_{2}^{1}$-DET" implies

$$
\operatorname{Con}\left(\mathrm{ZF}_{N_{0}}+\mathrm{AC}+\operatorname{Pr} \Delta_{2}^{1} \text {-DET Schema }+\forall x \in \mathbf{R} \exists M\right.
$$

\section{( $M$ is a standard model of ZFC containing $x)$ )}

and a fortiori the consistency of the weaker theory (where $\operatorname{Pr} \Delta_{2}^{1}$-DET is replaced by $\operatorname{Pr} \Delta_{2}^{1}$-DET) mentioned above.

REMARK. If $\mathrm{ZFC}+\sigma$ is an extension of ZFC by some further hypothesis for which we can construct canonical inner models (e.g. $\sigma=$ "There exists an inaccessible", $\boldsymbol{\sigma}=$ " $\forall x \in \mathbf{R}$ ( $x^{\#}$ exists)", $\boldsymbol{\sigma}=$ " $\exists$ a measurable cardinal", $\boldsymbol{\sigma}=$ " $\Delta_{2}^{1}$-DET" etc.), then we can calculate by similar techniques levels of lightface determinacy $\Gamma$-DET such that $\mathrm{ZFC}+\sigma+\mathrm{OD}-\mathrm{DET}$ is equiconsistent with $\mathrm{ZFC}+\sigma+\Gamma$-DET. If for instance $\sigma$ relativizes to the $L[x]$ 's for $x \in \mathbf{R}, \Gamma=\Delta_{2}^{1}$ will work again. If $\sigma=$ " $\forall x \in \mathbf{R}$ ( $x^{\#}$ exists)" a rough calculation will give that $\Gamma=\emptyset\left(\omega^{2}+1-\Pi_{1}^{1}\right)$ will work (instead of $L[x]$ one uses $L^{\#}[x]$, the smallest inner model of ZFC containing $x$ and closed under sharps for reals). Similarly for stronger hypotheses. In these cases the theory $\mathrm{ZFC}+\sigma+\mathrm{OD}-\mathrm{\sigma ET}$ can be quite strong. For instance we have that $\mathrm{ZFC}+$ $\forall x \in \mathbf{R}$ ( $x^{\#}$ exists) + OD-DET is stronger than $\Delta_{2}^{1}$-DET (boldface!).

5. Equivalent forms of determinacy. We can also use the ideas of $\S 3$ to find a simple proof of a generalization of Martin's Theorem that

$$
\mathrm{ZF}+\mathrm{DC} \vdash \Delta_{2 n}^{1}-\mathrm{DET} \Rightarrow \Sigma_{2 n}^{1}-\mathrm{DET} .
$$

To illustrate the idea let us give first a new proof that $\mathrm{ZF}+\mathrm{DC} \vdash \Delta_{2}^{1}$-DET $\Rightarrow$ $\Sigma_{2}^{1}$-DET (the argument clearly relativizes to produce its boldface form). Our general result is an abstraction of the basic idea in this argument.

Assume $\mathrm{ZF}+\mathrm{DC}+\Delta_{2}^{1}$-DET. We will show $\Sigma_{2}^{1}$-DET. Let $\varphi(\alpha)$ be a $\Sigma_{2}^{1}$ formula. If $L[x] \vDash$ "II has a winning strategy in $\{\alpha: \varphi(\alpha)\}$ " for some real $x$, then since this is a $\Sigma_{3}^{1}$ statement, II has (in the real world) a winning strategy in $\{\alpha: \varphi(\alpha)\}$ and we are done. So we can assume that for all reals $x, L[x] \vDash$ "II has no winning strategy in $\{\alpha: \varphi(\alpha)\} "$. So by Skolem-Löwenheim

$\forall x \in \mathbf{R} \exists \vartheta<\omega_{1} L_{\vartheta}[x] \vDash \mathrm{ZF}_{N}+$ "II has no winning strategy in $\{\alpha: \varphi(\alpha)\} "$

for some fixed large enough $N$. Thus let for each real $x$,

$$
\begin{array}{r}
\vartheta(x)=\text { least } \vartheta<\omega_{1} \text { such that } L_{\vartheta}[x] \vDash \mathrm{ZF}_{N}+ \\
\text { "II has no winning strategy in }\{\alpha: \varphi(\alpha)\} " .
\end{array}
$$


Consider then the following game $G$ :

$$
\begin{array}{ccc}
\underset{\alpha, x}{\text { I }} \quad & \text { II } & \text { I wins iff }\langle\alpha, \beta\rangle \in L_{\vartheta(x)}[x] \\
& \& L_{\vartheta(x)}[x] \vDash \varphi(\langle\alpha, \beta\rangle) .
\end{array}
$$

This is clearly a $\Delta_{2}^{1}$ game, so it is determined. If II has a winning strategy $\tau$, then as in $\S 3$ we can see easily that $L_{\vartheta(\tau)}[\tau] \vDash$ "II has a winning strategy in $\{\alpha: \varphi(\alpha)\}$ ", which is a contradiction. So assume that I has a winning strategy $\sigma$. We claim then that the strategy

$$
\sigma^{\prime}(\beta)=\alpha, \quad \text { where } \sigma(\beta)=(\alpha, x),
$$

is winning in the original game $\{\alpha: \varphi(\alpha)\}$. Indeed, if $\alpha, \beta, x$ are given by $(*)$, then $L_{\vartheta(x)}[x] \vDash \varphi(\langle\alpha, \beta\rangle)$. But $\varphi$ is $\Sigma_{2}^{1}$, so $\varphi(\langle\alpha, \beta\rangle)$ holds in the real world.

We state now our general theorem.

THEOREM 5.1. Assume ZF + DC. Let $\Gamma$ be a pointclass containing all the recursive pointsets, which is $\omega$-parametrized, has the prewellordering property and is closed under $\wedge, \vee, \exists^{\omega}, \forall^{\omega}$, recursive substitutions and $\exists^{\mathbf{R}}$. Then $\Delta$-DET $\Rightarrow \Gamma$-DET.

Here of course $\Delta=\Gamma \cap \check{\Gamma}$, where $\check{\Gamma}=\{\sim A: A \in \Gamma\}=$ the dual pointclass of $\Gamma$.

COROllary 5.2 (MARTin [Ma5]). For every $n \geqslant 0$,

$$
\mathrm{ZF}+\mathrm{DC}+\Delta_{2 n}^{1}-\mathrm{DET} \vdash \Delta_{2 n+2}^{1} \text { DET } \Rightarrow \Sigma_{2 n+1}^{1} \text {-DET }
$$

Martin's proof in [Ma5] adapts to show that

$$
\mathrm{ZF}+\mathrm{DC} \vdash \text { HYP-DET } \Rightarrow \text { IND-DET. }
$$

From 5.1 we also have a lightface version:

COROllaRY 5.3. ZF + DC $\vdash$ HYP-DET $\Rightarrow$ IND-DET.

Proof of The Theorem. Work in ZF + DC and assume $\Delta$-DET. Let $A \subseteq \mathbf{R}$ be a set in $\Gamma$. Let $W \subseteq \omega \times \mathbf{R}$ be universal for $\Gamma$ and such that for all $x \in \mathbf{R}, W^{x}=\{n$ : $W(n, x)\}$ is a $\Gamma(x)$-complete subset of $\omega$, in particular $W^{x} \notin \Delta(x)$. Let $\varphi: W \rightarrow$ ORD be a $\Gamma$-norm on $W$ and let $e_{0} \in \omega$ be such that $\alpha \in A \Leftrightarrow\left(e_{0}, \alpha\right) \in W$.

Assume II has no winning strategy in $A$. We shall produce a winning strategy for I in $A$.

If $\tau$ is a strategy for player II and I plays a real $\alpha$, denote by $\tau * \alpha$ the real that II plays following $\tau$ against $\alpha$. So we have $\forall \tau \exists \alpha(\langle\alpha, \tau * \alpha\rangle \in A)$. So in particular for each $x \in \mathbf{R}$,

$$
\forall \tau \leqslant{ }_{T} x \exists \alpha(\langle\alpha, \tau * \alpha\rangle \in A)
$$

We claim now that

$$
\forall x \exists n\left[(n, x) \in W \wedge \forall \tau \leqslant{ }_{T} x \exists \alpha\left(\varphi\left(e_{0},\langle\alpha, \tau * \alpha\rangle\right)<\varphi(n, x)\right)\right] .
$$

If not, then for some $x \in \mathbf{R}$ and all $n \in \omega$ :

$$
\forall \tau \leqslant_{T} x \exists \alpha\left(e_{0},\langle\alpha, \tau * \alpha\rangle\right)<_{\varphi}^{*}(n, x) \Rightarrow(n, x) \notin W,
$$

where

$$
w<_{\varphi}^{*} v \Leftrightarrow w \in W \wedge[v \notin W \vee \varphi(w)<\varphi(v)]
$$


so that since $\varphi$ is a $\Gamma$-norm, $<_{\varphi}^{*} \in \Gamma$. Therefore

$$
(n, x) \notin W \Leftrightarrow \forall \tau \leqslant T_{T} x \exists \alpha\left(\left(e_{0},\langle\alpha, \tau * \alpha\rangle\right)<_{\varphi}^{*}(n, x)\right),
$$

i.e. $W^{x} \in \check{\Gamma}(x)$ so $W^{x} \in \Delta(x)$, a contradiction.

By the Number Uniformization Theorem (see [Mo1, p. 202]), from (*) we have that there is a function $F: \mathbf{R} \rightarrow \omega$ with $\Delta$ graph such that

$$
\forall x\left[(F(x), x) \in W \wedge \forall \tau \leqslant_{T} x \exists \alpha\left(\left(e_{0},\langle\alpha, \tau * \alpha\rangle\right)<_{\varphi}^{*}(F(x), x)\right)\right] .
$$

Consider now the following game $G$ :

$$
\underset{\alpha, x}{\text { I }} \quad \text { II }_{\beta} \quad \text { I wins iff }\left(e_{0},\langle\alpha, \beta\rangle\right)<_{\varphi}^{*}(F(x), x) .
$$

Since $(F(x), x) \in W$ for all $x$, this is a $\Delta$ game so it is determined. If II has a winning strategy $\tau^{\prime}$ consider the strategy $\tau$ (for the original game $A$ ) which for each real $\alpha$ that I plays, it replies by playing $\tau * \alpha=\beta$, where $\beta=\tau^{\prime} *\left\langle\alpha, \tau^{\prime}\right\rangle$. Clearly $\tau \leqslant{ }_{T} \tau^{\prime}$ so

$$
\exists \alpha\left[\left(e_{0},\langle\alpha, \tau * \alpha\rangle\right)<_{\varphi}^{*}\left(F\left(\tau^{\prime}\right), \tau^{\prime}\right)\right] .
$$

Fix such an $\alpha$. If I plays $\alpha, \tau^{\prime}$ in $G$ then II replies by $\beta=\tau^{\prime} *\left\langle\alpha, \tau^{\prime}\right\rangle=\tau * \alpha$ and since he wins we have

$$
\neg\left[\left(e_{0},\langle\alpha, \tau * \alpha\rangle\right)<_{\varphi}^{*}\left(F\left(\tau^{\prime}\right), \tau^{\prime}\right)\right],
$$

a contradiction.

So I has a winning strategy $\sigma$ in $G$. We claim that forgetting about his extra move $x$, this is also a winning strategy for $A$. Indeed, if II plays $\beta$, I following $\sigma$ produces $\alpha$ and an auxiliary $x$ such that

$$
\left(e_{0},\langle\alpha, \beta\rangle\right)<_{\varphi}^{*}(F(x), x),
$$

in particular $\left(e_{0},\langle\alpha, \beta\rangle\right) \in W$, i.e. $\langle\alpha, \beta\rangle \in A$ and we are done.

REMARK. Note that in Theorem 5.1 the assumption that $\Gamma$ is closed under $\exists^{\mathbf{R}}$ is necessary. To see this consider $\Gamma=\Pi_{1}^{1}$.

\section{PART II. Hierarchy THEOREMS}

\section{Review of standard notions and statement of the main theorem.}

6.1. We say that a pointclass $\Gamma$ is almost adequate if it contains all the recursive pointsets and is closed under $\wedge, \vee$ and recursive substitutions. As in [Mo1] $\Gamma$ is adequate if it is also closed under bounded number quantification. (The class of $<\omega^{2}-\Pi_{1}^{1}$ sets (to be defined presently) is almost adequate but not adequate).

A coding of a pointclass $\Gamma$ is a surjection (the coding map, $\varphi$ ) of a subset $C$ of $\mathbf{R}$ (the set of codes) onto $\{A \subseteq \mathbf{R}: A \in \Gamma\}$. A coded pointclass is a pointclass $\Gamma$ together with a coding of $\Gamma$.

A coded pointclass is almost adequate if the underlying pointclass is almost adequate, and the various closure properties involved in the notion of "almost adequate pointclass" hold "uniformly in the codes". Thus, for example, if $\Gamma$ is an almost adequate coded pointclass, there will be recursive functions

$$
F_{1}: \mathbf{R} \times \mathbf{R} \rightarrow \mathbf{R}, \quad F_{2}: \omega \times \mathbf{R} \rightarrow \mathbf{R}
$$


such that:

(1) If $\alpha$ and $\beta$ are $\Gamma$-codes (for the sets $A, B$ respectively), then $F_{1}(\alpha, \beta)$ is a $\Gamma$-code for $A \cup B$.

(2) If $\alpha$ is a $\Gamma$-code for $A$ and $e$ is the Gödel number of the total recursive function $G: \mathbf{R} \rightarrow \mathbf{R}$, then $F_{2}(e, \alpha)$ is a $\Gamma$-code for $G^{-1}[A]$.

Similarly if we say that a coded pointclass has some other closure property (e.g. closure under complementation) we mean the underlying pointclass has the closure property "uniformly in the codes".

6.2. Now let $\Gamma$ be an almost adequate coded class. By a $\Gamma$-norm on a set $A$, we mean a map $\varphi: A \rightarrow \lambda$, such that (setting $\varphi(x)=\infty$ for $x \notin A$ ) the relations

$$
\begin{aligned}
& x \leqslant_{\varphi}^{*} y \Leftrightarrow x \in A \wedge \varphi(x) \leqslant \varphi(y), \\
& x<_{\varphi}^{*} y \Leftrightarrow x \in A \wedge \varphi(x)<\varphi(y)
\end{aligned}
$$

lie in $\Gamma$.

By a $\Gamma$-code for $\varphi$ we mean a pair $(\alpha, \beta)$ where $\alpha \Gamma$-codes (1), and $\beta \Gamma$-codes (2) when (1), (2) are coded in the obvious way as subsets of $\mathbf{R}$.

We say that a coded pointclass $\Gamma$ has the weak scale property if there is a recursive map $F: \omega \times \mathbf{R} \rightarrow \mathbf{R} \times \mathbf{R}$ and for each $\Gamma$-code $\alpha$ (of $A$, say) there exists a scale $\left\{\varphi_{n}^{\alpha}\right\}$ on $A$ such that $F(n, \alpha)$ is a $\Gamma$-code for $\varphi_{n}^{\alpha}$.

This definition is different from that of the scale property in [Mo1]. There, the

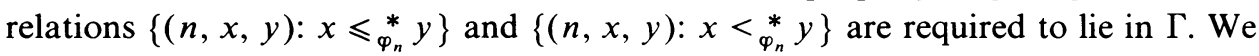
only require the pieces (for each fixed $n$ ) to lie in $\Gamma$ uniformly in the codes.

6.3. We now formulate the version of the " 3 rd Periodicity Theorem" which we will need for our proofs.

THEOREM (MOSCHOVAKIS [Mo1]). Let $\Gamma$ be an almost adequate coded pointclass with the weak scale property. Let $\alpha$ be a $\Gamma$-code for $A \subseteq \mathbf{R}$. Suppose that every game, whose payoff set has a $\Gamma$-code recursive in $\alpha$, is determined.

Then if player I wins the game A, I has a winning strategy recursive (uniformly in $\alpha$ ) in

$$
\left\{e:\{e\}^{\alpha} \text { is total and is a } \Gamma \text {-code for a game won by player } \mathrm{I}\right\} \text {. }
$$

(Here, $\{e\}^{\alpha}$ is the function partial recursive in $\alpha$ with Gödel number e.)

The theorem as formulated here is a slight variant of Theorem 6E.1 of [Mo1], and has "the same proof" as that theorem.

6.4. Let $\Gamma$ be a pointclass and $\mathscr{F}$ a collection of partial functions with domain and range a subset of $\mathbf{R}$. Then $\Gamma$ is closed under substitution by members of $\mathscr{F}$ if whenever $f \in \mathscr{F}$ and $A \in \Gamma$, then there is a $B \in \Gamma$ such that whenever $f(x)$ is defined,

$$
x \in B \leftrightarrow f(x) \in A .
$$

The particular class $\mathscr{F}^{*}$ that comes up in our applications is a subclass of the functions with $\Sigma_{3}^{0}$ graphs. Say that $f$ is nice $\Sigma_{3}^{0}$ if there is a $g: \mathbf{R} \rightarrow \omega$, partial with a $\Pi_{1}^{0}$ graph, and a partial recursive function $h: \omega \times \mathbf{R} \rightarrow \mathbf{R}$ such that $f(x) \cong$ $h(g(x), x)$.

6.5. We next recall the notion of an $\xi-\Pi_{1}^{1}$ set. Let $\xi$ be a countable ordinal. Set $\left\langle A_{\eta}: \eta\langle\xi\rangle\right.$ as a $\xi$-sequence of subsets of $\mathbf{R}$. Set $A_{\xi}=\varnothing$. Then the difference kernel 
of the sequence $\left\langle A_{\eta}: \eta<\xi\right\rangle$ is the set

$\left\{x\right.$ : The least $\eta$ such that $x \notin A_{\eta}$ is odd $\}$.

$A$ is $\xi-\Pi_{1}^{1}$ if $A$ is the difference kernel of a $\xi$ sequence of $\Pi_{1}^{1}$ sets. $A$ is $<\xi-\Pi_{1}^{1}$ if it is $\xi^{\prime}-\Pi_{1}^{1}$ for some $\xi^{\prime}<\xi$. If $\lambda$ is a limit ordinal, then $A$ is $(\lambda-1)-\Pi_{1}^{1}$ if $A$ is the difference kernel of a $\lambda$-sequence of $\Pi_{1}^{1}$ sets, $\left\langle A_{\xi}, \xi<\lambda\right\rangle$ such that $\bigcap_{\xi<\lambda} A_{\xi}=\varnothing$.

We shall need the corresponding lightface classes of $\xi-\Pi_{1}^{1}$ sets. We shall give a definition for $\xi=\omega^{2}$. An analogous definition can be given for any $\xi$ for which we know which are the "recursive" maps of $\xi$ onto $\omega$ (for example, any $\xi<\omega^{\omega}$ ). By a more complicated discussion, one can give a definition of $\xi-\Pi_{1}^{1}$ for any $\xi<\omega_{1}^{C K}$.

Let $A$ be a $\Pi_{1}^{1}$ subset of $\omega \times \mathbf{R}$. Let, for $\eta<\omega^{2}, A_{(\eta)}$ be defined as follows: if $\eta=\omega \cdot j+k, A_{(\eta)}=\left\{x:\left(2^{j} 3^{k}, x\right) \in A\right\}$. Let $B$ be the difference kernel of $\left\langle A_{(\eta)}, \eta\right.$ $\left\langle\omega^{2}\right\rangle$. Then as $A$ ranges over the $\Pi_{1}^{1}$ subsets of $\omega \times \mathbf{R}, B$ will range over the $\omega^{2}-\Pi_{1}^{1}$ subsets of $\mathbf{R}$. The notion of $<\omega^{2}-\Pi_{1}^{1}$ sets and $\left(\omega^{2}-1\right)-\Pi_{1}^{1}$ sets are defined similarly by analogy with the corresponding boldface definitions.

6.6. MAIN THEOREM. Let $\Delta$ be coded point class. We make the following assumptions on $\Delta$ :

(i) $\Delta$ is almost adequate;

(ii) $\Delta$ is closed under complementation;

(iii) $\Delta$ has the weak scale property;

(iv) The class $C$ of $\Delta$-codes is $\mathbf{R}$. Let $\left\{\alpha \mapsto S_{\alpha}\right\}$ be the coding map. Let $S=\{(\alpha, \beta)$ : $\left.\beta \in S_{\alpha}\right\}$. Then for some $N \geqslant 1, S$ is $\Delta_{N+1}^{1}$.

(v) If $N \geqslant 2$, we assume $\Sigma_{N}^{1} \subseteq \Delta$.

(Of course the closure properties of $\Delta$ expressed in (i), (ii), (iii) and (v) should hold "uniformly in the codes".)

Let $\varphi(x, y), \psi(x, y)$ be respectively $\Sigma_{N+1}^{1}$ and $\Pi_{N+1}^{1}$ formulas defining $S$. Let $\sigma_{1}$ be the sentence $(\forall x, y)[\varphi(x, y) \leftrightarrow \psi(x, y)]$. Let $\sigma_{2}$ be a sentence (of the language of set theory) expressing the various properties assumed of $\Delta$ (defining $\Delta$, using $\varphi$, in the evident way). We want $\sigma_{2}$ to mention the specific Gödel numbers of the recursive functions giving the "uniformities in the codes". Finally, we express " $\Delta$-DET" as a sentence of the language of set theory using the definition of $\Delta$ in terms of $\varphi$.

Then there is a game $G$, explicitly and uniformly definable from the data just described $(\Delta, \varphi, \psi$, etc.) such that: if $G$ is determined, then there is a countable transitive model of ZFC $+\sigma_{1}+\sigma_{2}+\Delta$-DET.

$G$ will lie in any pointclass $\Gamma$ with the following closure properties:

(i) $\Gamma$ is almost adequate;

(ii) $\Gamma$ contains all $\left(\omega^{2}-1\right)-\Pi_{1}^{1}$ sets;

(iii) $S \in \Gamma$;

(iv) $\Gamma$ is closed under substitution by nice $\Sigma_{3}^{0}$ partial functions. (In particular, $G$ will be a $\Delta_{N+1}^{1}$ game.)

6.7. The case $N \geqslant 2$ of the Main Theorem seems, at first glance, to be contrary to the main results of Part I of this paper. For the game $G$ will certainly be ordinal definable. And we showed in Part I that " $\mathrm{ZFC}+\Delta_{2}^{1}$-DET" has strictly greater consistency strength than "ZFC + OD-DET". 
If one analyzes this situation, one gets the following: "ZFC + OD-DET" cannot prove (except via an inconsistency) that there is a class $\Delta$ containing $\Sigma_{2}^{1}$ and satisfying the hypotheses of the Main Theorem, of which of course the only crucial one is the weak scale property.

Indeed, we know only a few ways of producing scales on pointclasses at $\Pi_{2}^{1}$ or beyond. They all require boldface determinacy hypotheses to work.

(1) Assuming $(\forall x \in \mathbf{R})$ ( $x^{\#}$ exists), one can put a scale on $\Pi_{2}^{1}$ sets (using the method of [MS]).

(2) Assuming $\Delta_{2 k}^{1}$-DET, one can show that the pointclasses $\Pi_{2 k+1}^{1}$ and $\Sigma_{2 k+2}^{1}$ have the scale property (cf. [Mo1, Theorems 6.C.2 and 6.C.3, "The Second Periodicity Theorem"]). It will follow that many pointclasses intermediate between $\Sigma_{2 k}^{1}$ and $\Delta_{2 k+2}^{1}$ will also have the scale property.

(3) If $\Gamma$ is an adequate pointclass, closed under continuous preimages, and with the scale property and every $\Gamma$-game is determined, then the class $\supset \Gamma$ has the scale property [Mo1, Exercise 6E.15]. For a discussion of the game quantifier see [Mo1, $\S 6 \mathrm{D}]$. This seems the most fruitful way, at present, to get interesting classes, to which the Main Theorem applies, beyond $\Delta_{2}^{1}$.

6.8. We next recall the basic closure properties of the $\xi-\Pi_{1}^{1}$ sets.

(1) Let $\left\langle A_{\eta}, \eta<\xi\right\rangle$ be a $\xi$-sequence of subsets of $\mathbf{R}$. Set $B_{\eta}=\bigcap_{\eta^{\prime} \leqslant \eta} A_{\eta^{\prime}}$ for $\eta<\xi$. Then $\left\langle A_{\eta}, \eta<\xi\right\rangle$ and $\left\langle B_{\eta}, \eta<\xi\right\rangle$ have the same difference kernel. Thus, without loss of generality, we may assume that we take the difference kernel only of decreasing $\xi$-sequences.

This makes contact with the discussion of the difference hierarchy in [Hf, §17]. The remaining facts we cite follow readily from that discussion.

(2) Let $\left\langle A_{\eta}, \eta<\xi\right\rangle$ be a decreasing $\xi$-sequence of $\Pi_{1}^{1}$ sets. If $\eta \geqslant \xi$, let $A_{\eta}=\varnothing$. Let $A$ be the difference kernel of $\left\langle A_{\eta}, \eta<\xi\right\rangle$. Then

$$
A=\bigcup_{\eta<\xi: \eta \text { even }}\left[A_{\eta}-A_{\eta+1}\right]
$$

Thus every $\xi-\Pi_{1}^{1}$ set is a countable union of finite Boolean combinations of $\Pi_{1}^{1}$ sets.

(3) If $A$ is $\Pi_{1}^{1}, A$ is $1-\Pi_{1}^{1}$.

(4) If $\xi<\vartheta$, every $\xi-\Pi_{1}^{1}$ set is $\vartheta-\Pi_{1}^{1}$.

(5) If $A$ is $\xi-\Pi_{1}^{1}, \sim A$ is $\xi+1-\Pi_{1}^{1}$.

(6) The class of $<\omega-\Pi_{1}^{1}$ sets is precisely the class of finite Boolean combinations of $\Pi_{1}^{1}$ sets.

(7) Let $\lambda$ be an additively closed limit ordinal. Then the $<\lambda-\Pi_{1}^{1}$ sets and the $(\lambda-1)-\Pi_{1}^{1}$ sets are closed under finite Boolean operations.

The obvious lightface analogues of (1)-(7) hold, with "the same proofs".

If the reader wishes to avoid tracking down the proof of (7), he can replace in Theorems $A$ and $B$ the class of $\left(\omega^{2}-1\right)-\Pi_{1}^{1}$ sets by a slightly larger class which is manifestly closed under finite Boolean operations. For example let $\Sigma_{1}^{0}\left(\Pi_{1}^{1}\right)$ denote the class of countable unions of finite Boolean combinations of $\Pi_{1}^{1}$ sets. Let $\Sigma_{1}^{0}\left(\Pi_{1}^{1}\right)$ 
denote the obvious lightface variant. Let $\Delta_{1}^{0}\left(\Pi_{1}^{1}\right)$ consist of those $A \subseteq \mathbf{R}$ such that $A$, $\sim A$ lie in $\Sigma_{1}^{0}\left(\Pi_{1}^{1}\right)$. Then if we replace (ii) in the Main Theorem by (ii'): $\Gamma$ contains all $\Delta_{1}^{0}\left(\Pi_{1}^{1}\right)$ sets, we get a variant which we can prove without reference to the results of [Hf, §17].

6.9. Finally, we wish to recall some results of Martin and Harrington.

(a) Theorem (Martin, Harrington [Ma1, Ma2 And H]). The following are equivalent:

(1) $\Pi_{1}^{1}$-DET.

(2) $\left(<\omega^{2}-\Pi_{1}^{1}\right)$-DET.

(3) $0^{\#}$ exists.

By relativization, this theorem has the following boldface corollary:

(b) COROLLARY. The following are equivalent:

(1) $\Pi_{1}^{1}$-DET,

(2) $\left(<\omega^{2}-\Pi_{1}^{1}\right)$-DET,

(3) $(\forall x \in \mathbf{R})\left(x^{\#}\right.$ exists $)$.

(c) THEOREM (MARTIN [Ma2]). The following are equivalent:

(1) $\left(\omega^{2}+1-\Pi_{1}^{1}\right)$-DET.

(2) $\left(<\omega^{2}+\omega^{2}-\Pi_{1}^{1}\right)$-DET.

(3) $0^{+}$exists.

Now ZFC $+0^{+}$exists $\vdash$ Con(ZFC $+\exists$ measurable cardinal $)$. Hence $\mathrm{ZFC}+\left(\omega^{2}\right.$ $\left.+1-\Pi_{1}^{1}\right)$-DET $\vdash$ Con $\left(\mathrm{ZFC}+\Pi_{1}^{1}\right.$-DET).

Our original version of the Main Theorem had the additional hypothesis that every game in $\Delta$ is determined. It was the theorem of Martin just cited that led us to look for a version with a "single game" determinacy hypothesis. Our first proof of a "single game" version used an unpublished "generic encoding" technique of Kunen. The present simpler proof evolved from suggestions of Martin, after he heard a talk on our earlier proof.

\section{The key game.}

7.1. Let $\Delta$ be as in the statement of the Main Theorem. Let the sentence $\sigma$ be the conjunction of $\sigma_{1}$ and $\sigma_{2}$ of that theorem and of a large finite subset of the axioms of ZFC (sufficiently large to carry out the usual arguments of descriptive set theory, as well as the usual theory of the $V_{\xi}$ 's).

Let $E \subseteq \mathbf{R}$ be $\left\{\alpha: S_{\alpha}\right.$ is a game won by player I $\}$. For $\xi \in \mathrm{ORD}$, we define $L_{\xi}[E]$, by induction on $\xi$, as follows: $L_{0}[E]=\varnothing ; L_{\eta+1}[E]$ consists of all first-order definable subsets of the structure $\left\langle L_{\eta}[E] ; \varepsilon, E \cap L_{\eta}[E]\right\rangle$; for $\lambda$ a limit ordinal, $L_{\lambda}[E]=\cup_{\eta<\lambda} L_{\eta}[E]$. We set $L[E]=\bigcup_{\xi \in \text { ORD }} L_{\xi}[E]$. Thus $L[E]$ can be characterized as the minimal transitive model, $M$, of ZFC, containing all the ordinals, and such that $E \cap M \in M$. Our desired transitive set model of $\mathrm{ZFC}+\sigma+$ $\Delta$-DET will eventually turn out to be $L_{\omega_{1}}[E]$. 
7.2. The following lemma is the place in our argument where we use our assumption that $\Delta$ has the weak scale property.

Lemma. $L[E] \vDash \sigma$. If $S_{\alpha}$ is determined for every $\alpha \in L[E] \cap \mathbf{R}$, then $L[E] \vDash \Delta$ DET.

(Note that our assumption is that $S_{\alpha}$ is determined in the real world. A priori, $S_{\alpha}$ need not be determined in $L[E]$.)

Proof. (1) Let $\alpha \in L[E] \cap \mathbf{R}$ be such that I wins $S_{\alpha}$ in $V$. The 3rd Periodicity Theorem $(\S 6.3)$, together with the definition of $E$, guarantees that a winning strategy for I in $S_{\alpha}$ will lie in $L[E]$.

(2) Claim. Let $N$ be as in the statement of the Main Theorem. (So $S$ is $\Delta_{N+1}^{1}$, and if $N \geqslant 2, \Sigma_{N}^{1} \subseteq \Delta$.) Let $A$ be a nonempty $\Pi_{N}^{1}$ subset of $\mathbf{R}$, which has $\Pi_{N}^{1}$-code lying in $L[E]$. Then $A \cap L[E] \neq \varnothing$.

Proof. If $N=1$, this follows from the Shoenfield Absoluteness Theorem. So let $N>1$. Let $G$ be the game which I wins iff the real played by I lies in $A$. That $G$ has a $\Delta$-code lying in $L[E]$ is clear from our closure assumptions on $\Delta$. Since $A \neq \varnothing, \mathrm{I}$ wins $G$. Hence, by (1), there is a winning strategy for $G$, lying in $L[E]$. So $A \cap L[E] \neq \varnothing$.

(3) As an immediate corollary of (2), $L[E]$ is $\Sigma_{N+1}^{1}$-correct (i.e., every $\Sigma_{N+1}^{1}$ assertion (with real parameters from $L[E]$ ) has the same truth value in $L[E]$ and in $V)$. Thus any $\Pi_{N+2}^{1}$ assertion (with parameters from $L[E]$ ), holding in $V$, holds also in $L[E]$.

(4) It follows that $\sigma_{1}$ holds in $L[E]$ and that for $\alpha \in \mathbf{R} \cap L[E]$,

$$
S_{\alpha}^{L[E]}=\{\beta \in L[E]: L[E] \vDash \varphi(\alpha, \beta)\}=S_{\alpha} \cap L[E] .
$$

Using this, it is easy to check that $\sigma_{2}$ also holds in $L[E]$. Hence, $L[E] \vDash \sigma$.

(5) Since $\Delta$ is almost adequate and closed under complements, the fact that $L[E] \vDash \Delta$-DET will follow from our determinacy hypothesis and the following

Claim. If $\alpha \in \mathbf{R} \cap L[E]$ and I wins $S_{\alpha}$ in $V$, then I wins $S_{\alpha}$ in $L[E]$.

Proof. By (1), there is a winning strategy for $\mathrm{I}$ in $S_{\alpha}, \tau$, lying in $L[E]$. In the presence of $\sigma$, the assertion " $\tau$ is a winning strategy for $\mathrm{I}$ in $S_{\alpha}$ " is a $\Pi_{N+1}^{1}$ assertion about $\tau$ and $\alpha$. Holding in $V$, it holds in $L[E]$, by (3).

7.3. We now give an informal description of the crucial game $G$. A more formal description will follow in $\$ 7.4$.

In $G$, player II will begin by answering a question about the "sharp" of the model $L[E]$. He will also provide "documentation" to support his description and will defend it against the challenges of player I. The game will be designed so that II can defeat any strategy for I by "telling the truth". Hence, if $G$ is determined, II must win. But I's opportunities for challenging II will be sufficiently abundant that II can only win by telling the truth.

In more detail, the game proceeds as follows. Let $\mathscr{L}_{1}$ be the language of set theory augmented by a one place predicate symbol $\underline{E}$, and an infinite stock of constant symbols $c_{0}, c_{1}, \ldots$

(1) I plays a sentence $\chi$ of $\mathscr{L}_{1}$.

(2) II plays a "truth value" for $\chi$ in $\{0,1\}$. (Say $1=$ "truth"; $0=$ "falsehood".) 
(3) Let $n$ be the least integer such that no $c_{i}$ appears in $\chi$ with $i \geqslant n$. I and II will "cooperatively produce" countable ordinals $\lambda_{0} \leqslant \lambda_{1} \leqslant \cdots \leqslant \lambda_{n}$. (The meaning of the phrase in quotes will be spelled out below.)

(4) II will play the satisfaction relation of some structure $\langle\omega ; R\rangle$, with $R$ a binary relation on $\omega$. For II to stay in the game, $\langle\omega ; R\rangle$ must be a well-founded model of $\sigma$. Let $\pi:\langle\omega, R\rangle \cong\langle M, \varepsilon\rangle$ be the transitive collapse map.

In addition, $M$ must satisfy the following requirements:

(a) $\lambda_{n} \in M$.

(b) Since $M \vDash \sigma$, we can form $L[E]^{M}$. We require $L_{\lambda_{n}}[E]^{M}$ to give $\chi$ the truth value predicted by II at stage (2), provided we interpret $\underline{E}$ by $E^{M} \cap L_{\lambda_{n}}[E]^{M}$, and interpret $c_{i}$ by $\lambda_{i}$ for $i<n$.

(5) At certain points during the game I may issue a "challenge" to II. At any given time, at most one challenge will be in effect. Thus each new challenge of I will have the side effect of revoking all earlier challenges.

When I issues a challenge, he points to a real $\alpha$ in $M$ (by naming the integer $j$ such that $\pi(j)=\alpha)$. $\alpha$ should be such that $M \vDash$ "I does not win $S_{\alpha}$ ". Players I and II begin playing $S_{\alpha}$ (on the side). If I issues a later challenge, this partial play of $S_{\alpha}$ is scrapped. For I to win, he must issue a last challenge, and win the play of the corresponding game.

This completes our sketch of the rules of $G$. A more complete (and more formal) description of $G$ will follow in a moment.

7.4. Let $\alpha$ be the element of $\mathbf{R}$ that records I's moves during a play of $G$, and let $\beta \in \mathbf{R}$ record II's moves.

We let $\alpha_{1}(n)=\alpha(3 n+1), \alpha_{2}(n)=\alpha(3 n+2), \alpha_{3}(n)=\alpha(3 n+3)$. Let $\beta_{1}, \beta_{2}, \beta_{3}$ be defined analogously from $\beta$. With respect to the outline in $\S 7.3$, these will serve the following functions:

$\alpha(0)$ will encode the $\chi$ of $(1) ; \beta(0)$ will encode II's assignment of a truth value to $x$.

$\alpha_{1}$ and $\beta_{1}$ will be reserved for the cooperative production of ordinals as mentioned in (3).

$\beta_{2}$ will be devoted to encoding the model $M$, and its satisfaction relation (cf. (4) of §7.3).

$\alpha_{2}$ will be the place where I records his challenges. Each $m$ such that $\alpha_{2}(m)>0$ represents a challenge by $I$.

The reals $\alpha_{3}$ and $\beta_{3}$ are used to record the moves of players I and II in the subsidiary games resulting from I's challenges.

7.5. The following clauses determine who wins $G$ if the play is the pair $(\alpha, \beta)$. The winner is determined by the first clause that applies.

(1) We fix a recursive Gödel numbering of $\mathscr{L}_{1}$. If $\alpha(0)$ is not the Gödel number of a sentence of $\mathscr{L}_{1}$, I loses.

(2) If $\beta(0)>1$, II loses.

(3) To each ordinal $\xi<\omega \cdot(n+1)$, we associate reals $\alpha_{1, \xi}, \beta_{1, \xi}$ as follows:

Let $\xi=\omega \cdot j+k$. Then $\alpha_{1, \xi}(m)=\alpha_{1}\left(2^{j} 3^{k} 5^{m}\right)$. $\beta_{1, \xi}$ is defined analogously from $\beta_{1}$. (Here $n$ is as defined in $\S 7.3(3)$.) 
Clause (3) will apply just in case for some $\xi<\omega \cdot(n+1)$, one of $\alpha_{1, \xi}, \beta_{1, \xi}$ does not code a countable ordinal. (For the usual coding of countable ordinals by reals, see [Mo1, §4.A.2].) In that case, let $\xi$ be least such that one of $\alpha_{1, \xi}, \beta_{1, \xi}$ does not code an ordinal. If $\alpha_{1, \xi}$ does not code an ordinal, II wins. Otherwise, I wins.

If clause (3) does not apply, we define countable ordinals $\lambda_{0}, \ldots, \lambda_{n}$ as follows:

$$
\lambda_{j}=\sup \left\{\left|\alpha_{1, \xi}\right|,\left|\beta_{1, \xi}\right|: \xi<\omega \cdot(j+1)\right\} .
$$

(Here if $\gamma \in \mathbf{R}$ codes an ordinal, $|\gamma|$ is that ordinal.)

Clause (4) will cause II to lose unless $\beta_{2}$ encodes a suitable model $M$. We let $\mathscr{L}_{2}$ be the language of set theory enriched by a constant $\underline{m}$ for each $m$ in $\omega$. II will lose unless all of the following happen:

(a) $\left\{m: \beta_{2}(m) \neq 0\right\}$ is the set of Gödel numbers of sentences in some complete consistent theory in the language $\mathscr{L}_{2}$.

(b) If $\theta$ is a sentence of $\mathscr{L}_{2}$, let $\ulcorner\boldsymbol{\theta}\urcorner$ be its Gödel number. Let $R=\{(i, j)$ : $\left.\left.\beta_{2}(\underline{i} \in \underline{j}\urcorner\right)>0\right\}$. Interpret $\underline{i}$ by $i$ for $i \in \omega$. Then for any sentence $\theta$ of $\mathscr{L}_{2},\langle\omega$; $R\rangle \vDash \theta$ iff $\beta_{2}\left({ }^{\top} \theta^{\top}\right)>0$.

(c) $\langle\omega ; R\rangle$ is a well-founded model of the sentence $\sigma$ of $\S 7.1$.

(d) Let $M$ be the transitive realization of $\langle\omega, R\rangle$. Then the $\lambda_{0}, \ldots, \lambda_{n}$ lie in $M$.

(e) Since $M \vDash \sigma$, we can carry out the construction of $L_{\lambda_{n}}[E]$ within $M$. Let $N$ be $L_{\lambda_{n}}[E]^{M}$. Interpret $\mathscr{L}_{1}$ in $N$ by interpreting $c_{i}$ by $\lambda_{i}$ for $i<n$ (and by 0 if $i \geqslant n$ ). Interpret $\underline{E}$ by $E^{M} \cap N$. Let $\chi$ be the sentence of $\mathscr{L}_{1}$ with Gödel number $\alpha(0)$. Then $N \vDash \chi$ iff $\beta(0)=1$.

(5) We now describe precisely the challenge mechanism.

(a) I loses unless for some $m, \alpha_{2}(m) \geqslant 1$ but $(\forall j>m)\left(\alpha_{2}(j)=0\right)$.

If clause (5)(a) does not apply, there is clearly a unique last $m$ such that $\alpha_{2}(m)>0$. Let $\alpha_{2}(m)=j+1$. Let $\pi: \omega \rightarrow M$ be as in $\S 7.3(4)$.

(b) I loses unless $\pi(j)$ is a $\gamma \in \mathbf{R}$, and $M \vDash$ "I does not win the game $S_{\gamma}$ ".

(c) Define a real $\delta \in \mathbf{R}$ by:

$$
\delta(2 i)=\alpha_{3}(m+i) ; \quad \delta(2 i+1)=\beta_{3}(m+i) \quad(\text { for } i \in \omega) .
$$

Then I wins iff $\delta \in S_{\gamma}$. Otherwise, II wins.

This completes our formal specification of the game $G$. The reader is advised to convince himself that clause (5) captures our informal discussion of challenges.

7.6. The device of "cooperatively producing ordinals" used in clause (3) of $\S 7.5$ goes back to an unpublished proof of Solovay (see however [Ke2]) that AD implies that $\omega_{1}$ is measurable. The following lemma, due to Martin in a slightly different context, is the key to the present application of this device.

LEMMA. Let $\tau$ be a winning strategy for I in $G$. Let $n$ be determined from I's first move according to $\tau$ as in $\S 7.3(3)$. Let $\delta_{0}<\delta_{1}<\cdots<\delta_{n}$ be countable ordinals which are limit points of ordinals admissible in $\tau$. Then there is a real $\gamma$ such that whenever $(\alpha, \beta)$ is a play of $G$ in which I plays according to $\tau, \beta(0) \leqslant 1$ and $\beta_{1}=\gamma$, then in the resulting play, none of clauses (1) through (3) of $\$ 7.5$ will apply. Moreover the ordinals $\lambda_{0}, \ldots, \lambda_{n}$ determined at the end of clause (3) will take on the values $\delta_{0}, \ldots, \delta_{n}$. 
Proof. Let $\left\langle\theta_{i}, i<\omega \cdot(n+1)\right\rangle$ be an increasing sequence of $\tau$-admissibles such that

$$
\delta_{j}=\sup \left\{\theta_{i}: i<\omega \cdot(j+1)\right\} \quad \text { for } j \leqslant n .
$$

We choose $\gamma$ so that whenever $\beta_{1}=\gamma, \beta_{1, \mathrm{i}}$ codes the ordinal $\theta_{i}$.

Suppose then that I plays according to the strategy $\tau$, and II plays so that $\beta(0) \leqslant 1$ and $\beta_{1}=\gamma$. Let the resulting sequence of I's moves be $\alpha$. In deciding who wins, clause (1) does not apply (since $\tau$ wins for I), and clause (2) does not apply (since $\beta(0) \leqslant 1$ ). If clause (3) applied, then since $\tau$ wins for I, some $\beta_{1, i}$ fails to code an ordinal $(i<\omega \cdot(n+1))$, contrary to our choice of $\gamma\left(=\beta_{1}\right)$.

It remains to see that $\lambda_{j}=\delta_{j}$ for $j \leqslant n$. For this it suffices to show:

Claim. $\left|\alpha_{1, i}\right|<\boldsymbol{\theta}_{i+1}$ for $i<\omega \cdot(n+1)$.

Let $y$ be a real in which $\tau$ is recursive and such that $\theta_{i}<\omega_{1}^{y} \leqslant \theta_{i+1}$. ( $y$ can be obtained by interweaving $\tau$ and a suitably generic code for $\theta_{i}$.)

Consider the set of reals $T=\left\{z:(\exists \alpha, \beta)\left(\beta(0) \leqslant 1 \wedge(\forall j \leqslant i)\left(\beta_{1, j}\right.\right.\right.$ codes an ordinal $\leqslant \theta_{i}$ ), and $\alpha$ is the real I obtains by playing the strategy $\tau$ against $\beta$ and $\left.\left.z=\alpha_{1, i}\right)\right\}$.

Then $T$ is clearly a $\Sigma_{1}^{1}(y)$ set of reals. Since $\tau$ wins for $\mathrm{I}$, any $z \in T$ codes an ordinal. Hence, by the boundedness theorem (cf. [Mo1, §4A.4]) any $z \in T$ codes an ordinal $<\omega_{1}^{y} \leqslant \theta_{i+1}$. In particular if $\alpha$ is as in our claim, $\alpha_{1, i} \in T$. So the claim and hence the lemma is proved.

In an entirely analogous fashion one can show:

LeMma. Let $\tau$ be a winning strategy for player II in $G$. Let $\left\langle\delta_{i}, i \in \omega\right\rangle$ be a strictly increasing sequence of countable ordinals such that each $\delta_{i}$ is a limit of admissibles in $\tau$. Then there is a real $\gamma$ so that whenever I plays $\alpha$ such that $\alpha(0)$ is the Gödel number of a sentence of $\mathscr{L}_{1}$ and $\alpha_{1}=\gamma$, and II plays according to $\tau$, then in the resulting play, clauses (1) through (3) do not apply, and $\lambda_{i}=\delta_{i}$ for $0 \leqslant i \leqslant n$.

\subsection{LemMA. Player I does not win G.}

Proof. Suppose to the contrary that I wins $G$ via the strategy $\tau$. We shall construct a play for II in which he defeats $\tau$, getting the desired contradiction. As we indicated previously, the basic idea behind the proof of the lemma is that II can defeat I by "telling the truth."

Let $\chi$ be the sentence of $\mathscr{L}_{1}$ that I plays on his first move, and let $n$ be determined from $\chi$, as usual. Let $\delta_{1}, \ldots, \delta_{n}$ be the first $n+1$ limits of $\tau$-admissible ordinals. II sets $\beta(0)=1$ (respectively 0 ) according to whether $L_{\delta_{n}}[E] \vDash \chi$ or not when $\underline{E}$ is interpreted by $E \cap L_{\delta_{n}}[E]$, and $c_{0}, \ldots, c_{n-1}$ by $\delta_{0}, \ldots, \delta_{n-1}$. Let $\gamma_{1}$ be the real provided by the first lemma in $\$ 7.6$ such that if $\beta_{1}=\gamma_{1}$, then $\lambda_{0}, \ldots, \lambda_{n}$ will take on the values $\delta_{0}, \ldots, \delta_{n}$. II will choose $\beta$ so that $\beta_{1}$ does equal $\gamma_{1}$.

Let $\theta$ be a limit ordinal $>\omega$, such that $V_{\theta} \vDash \sigma$. Let $M_{1}$ be a countable elementary submodel of $V_{\theta}$ with $\delta_{n} \cup\left\{\delta_{n}\right\} \subseteq M_{1}$. Let $M_{0}$ be the transitivization of $M_{1}$. Since $M_{0}$ is countable, we can clearly choose $\gamma_{2}$ so that if $\beta_{2}=\gamma_{2}$, then $M$ will equal $M_{0}$. II will choose $\beta_{2}$ so that $\beta_{2}=\gamma_{2}$. It is clear that no matter how II chooses $\beta_{3}$, if I plays according to $\tau$, then the resulting play of $G$, II will not lose according to clauses (1) through (4). 
In all plays of $G$ considered until this lemma is proved, I will play according to the strategy $\tau$, and II will choose $\beta(0), \beta_{1}$ and $\beta_{2}$ as previously indicated.

We say that a finite sequence $s$ of integers is special if whenever $\beta_{3}(i)=s(i)$ for $i<$ length $(s)$, then $\alpha_{2}($ length $(s))>0$.

Claim 1. Special sequences exist.

Proof. Let II play $\beta_{3}(m)=0$, all $m$. Since I wins, $\alpha_{2}(m)>0$ for some $m$. But $\alpha_{2}(m)=\alpha(3 m+2)$ is determined by $\tau$ without knowledge of $\beta_{3}(j)=\beta(3 j+3)$ for $j \geqslant m$. So if $s$ is a sequence of 0 's of length $m, s$ is special.

Say that $s$ is maximal special if $s$ is special and no proper end extension of $s$ is special.

Ciaim 2. Maximal special sequences exist.

Deny. Then we can define a sequence $\left\langle s_{i}, i<\omega\right\rangle$, where $s_{i}$ is special of length $m_{i}$ and $m_{0}<m_{1}<m_{2} \cdots$ and $s_{i+1}$ end-extends $s_{i}$. The union of the $s_{i}$ 's is an element of $\mathbf{R}, \gamma_{3}$. Have II play $\beta$ such that $\beta_{3}=\gamma_{3}$. Since $\beta_{3}$ extends $s_{i}, \alpha_{2}\left(m_{i}\right)>0$. So I loses, since $\alpha_{2}(m)>0$ for infinitely many $m$. This contradicts $\tau$ 's being a winning strategy for $G$.

Fix a maximal special sequence, $s_{0}$, of length $m_{0}$. From now on, we only consider plays of II in which $\beta_{3} \uparrow m_{0}=s_{0}$. The value of $\alpha_{2}\left(m_{0}\right)$ clearly does not depend on the value of $\beta_{3}(j)$ for $j \geqslant m_{0}$. Say $\alpha_{2}\left(m_{0}\right)=k+1$. Also in a play of $G$ in which $\beta_{3} \uparrow m_{0}=s_{0}$, we have $\alpha_{2}(m)=0$ for $m \geqslant m_{0}+1$. (If not, $\beta_{3} \uparrow m$ would be a special sequence which is a proper end extension of $s_{0}$, contrary to the choice of $s_{0}$.)

We now define a strategy $\tau^{*}$ for I. Let $t$ be a finite sequence of integers. Then $\tau^{*}(t)$ is the value $\alpha_{3}$ (length $(t)+m_{0}$ ) takes when $\beta_{3} \uparrow m_{0}+$ length $(t)$ is $\hat{s_{0}} t$.

The significance of $\tau^{*}$ is as follows. Let $(\alpha, \beta)$ be any play of $G$ in which I plays according to $\tau$, and II plays a $\beta$ with $\beta(0), \beta_{1}, \beta_{2}$ as described above and $\beta_{3} \uparrow m_{0}=s_{0}$. Then clauses (1) through (4) and clauses (5)(a) and (5)(b) of $\S 7.5$ will not apply. The last $m$ such that $\alpha_{2}(m)>0$ will be $m_{0}$. Let $\pi: \omega \rightarrow M$ be the canonical enumeration determined by $\beta_{2}$. Since clause (5)(b) does not apply (since $\tau$ is a winning strategy for I), $\pi(k)$ is a real, $\gamma_{2}$, in $\mathbf{R} \cap M$, and $M \vDash$ "I does not win $S_{\gamma_{2}}$ ". In clause (5)(c) a certain play of $S_{\gamma_{2}}$ is examined. In this play of $S_{\gamma_{2}}$, I plays according to $\tau^{*}$.

Let $\pi_{1}: M \rightarrow V_{\theta}$ be the inverse of the transitive collapse of $M_{1}$. Then $\pi_{1}$ is an elementary embedding, so $V_{\theta} \vDash$ "I does not win $S_{\gamma_{2}}$ ". It follows that there is a real $\beta^{*}$ such that if II plays $\beta^{*}$ and I plays according to $\tau^{*}$, I will not win $S_{\gamma_{2}}$.

Now let $\beta_{3}$ be chosen equal to $\hat{s_{0}} \beta^{*}$, and let II choose $\beta(0), \beta_{1}, \beta_{2}$ as previously indicated. Let I play according to $\tau$. Then in the play of $S_{\gamma_{2}}$ evaluated in (5)(c), II plays $\beta^{*}$ and I plays according to $\tau^{*}$. By our choice of $\beta^{*}$, II will win this play of $S_{\gamma_{2}}$. So II wins the play of $G$. But this contradicts $\tau$ being a winning strategy for I in $G$.

7.8. Assume now that $G$ is determined. By Lemma 7.7, II wins. We fix a winning strategy, $\tau$, for II once and for all. In all the plays of $G$ considered from now on, II will play according to the strategy $\tau$. Our next lemma has the following intuitive content. By exploiting the ability to challenge II, player I can insure that player II "tells the truth". 
LEMma. Let $\chi$ be a sentence of $\mathscr{L}_{1}$. Let $\left\langle\delta_{i} ; i\langle\omega\rangle\right.$ be an increasing sequence of countable ordinals, each of which is a limit of $\tau$-admissible ordinals. Let $X$ be $a$ countable subset of $\mathbf{R}$. Then there is a play $(\alpha, \beta)$ of $G$ such that:

(1) $\left.\alpha(0)={ }^{r} \chi\right\urcorner$.

(2) For $i \leqslant n, \lambda_{i}=\delta_{i}$.

(3) Let $z \in X \cap M$. Then if II does not win $S_{z}$, then $M \vDash$ "I wins $S_{z}$ ".

(4) II plays according to $\tau$.

Proof. Select $\gamma_{1}$ by the second lemma of $\$ 7.6$ such that if $\left.\alpha(0)={ }^{\ulcorner} \chi\right\urcorner$ and $\alpha_{1}=\gamma_{1}$, then (2) will hold. From now till the end of the proof of this lemma, all plays of $G$ considered will have $\left.\alpha(0)={ }^{r} \chi\right\urcorner$ and $\alpha_{1}=\gamma_{1}$.

Let $X_{1}=\left\{\gamma \in X\right.$ : II does not win $\left.S_{\gamma}\right\}$. If $X_{1}$ is empty, the lemma is trivial. So assume $X_{1} \neq \varnothing$.

We fix an enumeration, $\left\langle\left(n_{i}, x_{i}\right) ; i \in \omega\right\rangle$, of $\omega \times X_{1}$. We are going to construct $\alpha_{2}, \alpha_{3}$ in stages. At the beginning of the $i$ th stage, we will have determined $\alpha_{2} \uparrow m_{i}$; $\alpha_{3} \uparrow m_{i}$. We will have $m_{i+1}>m_{i}$. Thus after countably many stages, we will have determined $\alpha_{2}$ and $\alpha_{3}$ and thus a play of $G$. We will show then that this play of $G$ satisfies the conditions of the lemma. (To start things off, we will take $m_{0}=0$.)

We will say that the $i$ th stage is terminated if one of the following happens:

(i) $\beta_{2}\left(\left\ulcorner n_{i} \in \mathbf{R}\right\urcorner\right)=0$.

(ii) $\beta_{2}\left(\left\ulcorner\overline{\mathrm{I}}\right.\right.$ does not win the game with $\Delta$-code $\left.\left.n_{i}\right\urcorner\right)=0$.

(iii) For some $r$ and $k$ with $x_{i}(r)=k, \beta_{2}\left(\left\ulcorner(r, \bar{k}) \in n_{i}^{\urcorner}\right)=0\right.$.

Thus the $i$ th stage is never terminated iff $\pi\left(n_{i}\right)=\overline{x_{i}}$ and $M \vDash$ "I does not win the game with $\Delta$-code $x_{i} "$.

Suppose we are at the beginning of the $i$ th stage. A pair of finite sequences $(s, t)$ is a terminator for the $i$ th stage if:

(1) $s, t \in{ }^{<\omega} \omega$.

(2) length $(s)=$ length $(t)$.

(3) $s(0)=n_{i}+1$.

(4) For $0<j<$ length $(s), s(j)=0$.

(5) If $\alpha_{2}$ extends $\alpha_{2} \uparrow \hat{m_{i}} s$ and $\alpha_{3}$ extends $\alpha_{3} \uparrow \hat{m_{i}} t$, then for some $j<\operatorname{length}(s)$, $\beta_{2}(j)=0$ and this is sufficient for the $i$ th stage to be terminated (under one of (i) through (iii) above).

Claim. A terminator for the $i$ th stage exists (for every $i$ ).

Granted the claim we prove the lemma as follows. We construct $\alpha_{2}$ and $\alpha_{3}$ stage by stage as indicated previously. To get from stage $i$ to stage $i+1$, we pick a terminator $\langle s, t\rangle$ for stage $i$ and set: $m_{i+1}=m_{i}+\operatorname{length}(s) ; \alpha_{2} \uparrow m_{i+1}=\alpha_{2} \uparrow \hat{m_{i}} s$; $\alpha_{3} \uparrow m_{i+1}=\alpha_{3} \uparrow \hat{m_{i}} t$. At the end of the construction we will have determined $\alpha_{2}$ and $\alpha_{3}$ and hence a play $(\alpha, \beta)$ of $G$ in which II plays according to $\tau$. By previous remarks this play will satisfy (1) and (2) of the lemma. We must show that it satisfies (3).

Then let $\gamma \in X \cap M$ such that II does not win the game $S_{\gamma}$. Then $\gamma \in X_{1}$, so we can find $i$ such that $\pi\left(n_{i}\right)=\gamma$, and $x_{i}=\gamma$. Since stage $i$ was terminated, we must have $M \vDash$ "I wins $S_{\gamma}$ ". 
It remains to prove the claim. We suppose the claim is false and show how I can defeat $\tau$ (contradicting the fact that $\tau$ is a winning strategy for II).

I will play $\alpha(0), \alpha_{1}$ as previously indicated. He will play $\alpha_{2} \uparrow m_{i}$ as previously selected. He will set $\alpha_{2}\left(m_{i}\right)=n_{i}+1$ and $\alpha_{2}(j)=0$ for $j>m_{i}$. In any play we consider from now on $\alpha_{2}$ will be as just described. Moreover $\alpha_{3} \uparrow m_{i}$ will be as decided before stage $i$.

In any such play, $\pi\left(n_{i}\right)=x_{i}$ and $M \vDash$ "I does not win $S_{x_{i}}$ ". Thus in any such play, clauses (1) through (4), and clauses (5)(a) and (5)(b) of $\$ 7.5$ will not apply, and the winner of $G$ will be determined by who wins the "game within the game" $G_{x_{i}}$.

We define a strategy $\tau^{*}$ for II as follows. Let $s$ be a finite sequence of integers of length $m$. Suppose that I plays $\alpha_{3}$ extending $\alpha_{3} \uparrow \hat{m_{0}} s$. Then the value of $\beta_{3}(m-1)$ is well defined (since $\beta_{3}(m-1)=\beta(3 m)$, and for $j \geqslant m \alpha_{3}(j)=\alpha(3 j+3)$, which is not seen by $\tau$ when deciding $\beta(3 m)$ ). We take this value to be $\tau^{*}(s)$. The significance of $\tau^{*}$ is that in the "game within the game", II plays according to $\tau^{*}$. Now since $x_{i} \in X_{1}$, II does not win $S_{x_{i}}$. So there is an $\alpha^{*}$ such that if I plays $\alpha^{*}$ and II plays according to $\tau^{*}$, then I will win $S_{x_{i}}$. We now define $\alpha_{3}\left(m_{i}+j\right)$ to be $\alpha^{*}(j)$. Then if I plays $\alpha$, in the "game within the game" he will play $\alpha^{*}$, and win $G$ according to clause (5)(c). This contradicts the fact that $\tau$ wins $G$ for II, and proves our claim. This completes the proof of Lemma 7.8.

7.9. LeMma. Let $\chi$ be a sentence of $\mathscr{L}_{1}$. Let $n$ be least such that no $c_{i}$ appears in $\chi$ for $i \geqslant n$. Let $\delta_{0}<\delta_{1}<\cdots<\delta_{n}$ be ordinals which are limit points of the class of $\tau$ admissible ordinals. Interpret $\mathscr{L}_{1}$ in $L_{\delta_{n}}[E]$ by letting $c_{i}$ denote $\delta_{i}$ for $i<n$, and letting $\underline{E}$ denote $E \cap L_{\delta_{n}}[E]$. Then $\tau\left(\left\langle\left\ulcorner\chi^{\urcorner}\right\rangle\right)=1\right.$ iff $L_{\delta_{n}}[E] \vDash \chi$.

Proof. An easy Skolem-Löwenheim argument shows that we may as well assume the $\delta_{i}$ 's are countable. We apply Lemma 7.8, taking $X$ to be $\mathbf{R} \cap L_{\delta_{n}}[E]$. We get a play of $G$ in which II plays according to $\tau$, and $\lambda_{i}=\delta_{i}$ for $i \leqslant n$. Let $M$ be the transitive model of $\sigma$ determined by $\beta_{2}$. It suffices to prove $E^{M} \cap L_{\delta_{n}}[E]^{M}=E \cap$ $L_{\delta_{n}}[E]$ and $L_{\delta_{n}}[E]^{M}=L_{\delta_{n}}[E]$. For then $\tau\left(\left\langle\left\ulcorner\chi^{\urcorner}\right\rangle\right)\right.$will correctly predict the truth value of $\chi$ in $\left\langle L_{\delta_{n}}[E] ; \varepsilon, E \cap L_{\delta_{n}}[E]\right\rangle$ (since $\tau$ wins for II, and hence II does not lose by clause (4)).

Suppose not. We shall derive a contradiction. Let $\xi \leqslant \delta_{n}$ be least such that $\left\langle L_{\xi}[E] ; \varepsilon, E \cap L_{\xi}[E]\right\rangle$ gets different values in $V$ and in $M$. Then for some ordinal $\eta$, $\xi=\eta+1, L_{\xi}[E]=L_{\xi}[E]^{M}$ but $E \cap L_{\xi}[E] \neq E^{M} \cap L_{\xi}[E] .\left(L_{\xi}[E]=\right.$ all first-order definable subsets of $\left\langle L_{\eta}[E] ; \varepsilon, E \cap L_{\eta}[E]\right\rangle=$ all first-order definable subsets of $\left\langle L_{\eta}[E]^{M} ; \varepsilon, E^{M} \cap L_{\eta}[E]^{M}\right\rangle=L_{\xi}[E]^{M}$.)

Fix $x \in L_{\xi}[E]$ such that $x \in E \leftrightarrow x \notin E^{M}$. Then $x \in L_{\xi}[E]^{M} \subseteq M$ and $x \in X$ $=L_{\delta_{n}}[E] \cap \mathbf{R}$.

Suppose first that $x \in E$. Then I wins $S_{x}$. So II does not win $S_{x}$. By (3) of Lemma 7.8, $M \vDash$ "I wins $S_{x}$ ". I.e., $x \in E^{M}$, a contradiction.

Suppose next that $x \notin E$. We can find $x^{*}$ recursive in $x$ such that $V$ and $M$ both think

$$
S_{x^{*}}=\left\{\hat{n} y: n \in \omega \text { and } y \notin S_{x}\right\}
$$


Then clearly $x^{*} \in X \cap M$. Since $x \notin E$, I does not win $S_{x}$. So II does not win $S_{x^{*}}$. By (3) of Lemma 7.8, $M \vDash$ "I wins $S_{x^{*}}$ ". But then $M \vDash$ "I does not win $S_{x}$ ". So $x \notin E_{M}$, a contradiction.

7.10. Lemma. (1) Let $\delta$ be a limit of $\tau$-admissibles. Then $L[E] \vDash " \delta$ is strongly inaccessible".

(2) Let $\delta_{1}<\delta_{2}$ be limits of $\tau$-admissibles. Then $L_{\delta_{1}}[E] \prec L_{\delta_{2}}[E]$.

Proof. We prove part of (1) and leave the remainder of (1) and (2) for the reader. For (1), we must show: (1a) $L[E] \vDash$ " $\delta$ is regular" and (1b) $L[E] \vDash " \delta$ is a strong limit cardinal." To prove (1a) it suffices, by the reflection principle, to show that if $\delta^{\prime}$ is a limit of $\tau$ admissibles greater than $\delta$, then $L_{\delta^{\prime}}[E] \vDash$ " $\delta$ is regular". By Lemma 7.9 , if this holds for one pair $\delta, \delta^{\prime}$ it holds for all such pairs. It suffices to take $\delta=\omega_{1}$ and $\delta^{\prime}$ such that $L_{\delta^{\prime}}[E]$ is a $\Sigma_{3^{-}}$-elementary submodel of $L[E]$.

REMARK. It follows from Lemma 7.9 that $\langle L[E] ; \varepsilon, E\rangle$ has a proper class of indiscernibles. Hence $\langle L[E] ; \varepsilon, E\rangle$ has a "sharp". Using the fact that $\{\eta: \eta$ is a limit of $\tau$-admissibles $\}$ is club in $\omega_{1}$, we can show this sharp is $\{\chi: \tau(\langle\ulcorner\chi\urcorner\rangle)=1\}$.

\subsection{Lemma. Let $x \in L[E] \cap \mathbf{R}$. Then $S_{x}$ is determined.}

Proof. Suppose not. We shall derive a contradiction. Let $x$ counterinstance the lemma. Let $\delta_{0}$ be a limit of $\tau$-admissibles, such that $\delta_{0}$ is countable and $x \in L_{\delta_{0}}[E]$. We apply Lemma 7.8 , taking $X=\mathbf{R} \cap L_{\delta_{0}}[E]$. Let $(\alpha, \beta)$ be the play guaranteed by Lemma 7.8. Let $x^{*}$ be as in the proof of Lemma 7.9.

Now the proof of Lemma 7.9 shows $L_{\delta_{0}}[E]^{M}=L_{\delta_{0}}[E]$. So $x, x^{*} \in M$. Since $S_{x}$ is undetermined, II does not win $S_{x}$ or $S_{x^{*}}$. Hence, by (3) of Lemma 7.8 $M$ ₹"I wins both $S_{x}$ and $S_{x^{*}}$ ". But clearly $M \vDash$ "I wins $S_{x^{*}}$ iff II wins $S_{x}$ ". So $M \vDash$ "Both I and II win $S_{x}$ ". This is our desired contradiction.

7.12. By Lemmas 7.11 and 7.2, $L[E] \vDash \sigma+\Delta$-DET. By Lemma 7.10, it follows easily that $L_{\omega_{1}}[E] \vDash$ "ZFC $+\sigma+\Delta$-DET". To complete the proof of the Main Theorem, it suffices to show $G$ lies in $\Gamma$. Since the class of $\left(\omega^{2}-1\right)-\Pi_{1}^{1}$ sets is closed under complements and finite unions, our claim that $G$ lies in $\Gamma$ will follow from the following observations.

(1) Each of clauses (1) through (4), and clauses (5)(a), (5)(b) are $\left(\omega^{2}-1\right)-\Pi_{1}^{1}$.

Indeed, clauses (1), (2), (4), (5)(a), (5)(b) are obviously in the class of finite Boolean combinations of $\Pi_{1}^{1}$ sets. That clause $(3)$ is $\left(\omega^{2}-1\right)-\Pi_{1}^{1}$ we leave as an exercise for the reader.

Finally, it is easy to see that the map which sends $(\alpha, \beta)$ to $\delta$ as in (5)(c) (defined just in case $m$ is defined by clause (5)(a)) is a nice $\Sigma_{3}^{0}$ partial function. The fact that $G$ is in $\Gamma$ should now be clear.

\section{Some concrete hierarchy theorems within $\Delta_{2}^{1}$.}

8.1. In this section, we record, without proofs, some concrete hierarchy theorems which can be proved using the results and techniques of the earlier sections. Our first result is a slight improvement of the result already mentioned in $\$ 6$.

THEOREM. ZFC $+\left(\left(\omega^{2}-1\right)-\Pi_{1}^{1}\right)$-DET $\vdash \operatorname{Con}\left(\mathrm{ZFC}+\Pi_{1}^{1}\right.$-DET $)$. 
REMARKS ON THE PROOF. We just apply the Main Theorem with $\Gamma$ the class of $\left(\omega^{2}-1\right)-\Pi_{1}^{1}$ sets and $\Delta$ the class of $<\omega^{2}-\Pi_{1}^{1}$ sets. The key point is that $\Delta$ has the weak scale property. This is a result of Steel [St2].

8.2. We wish next to describe a general procedure for constructing $\Delta_{2}^{1}$ subsets of R. Our procedure uses the relativized Jensen hierarchy for $L[x]$ (cf. $[\mathbf{J}]$ ). Let $x \in \mathbf{R}$. By induction on $\xi \in \mathrm{ORD}$, we define $J_{\xi}[x] ; J_{0}[x]=\varnothing ; J_{\eta+1}[x]$ is the rudimentary closure of $J_{\eta}[x] \cup\left\{x, J_{\eta}[x]\right\}$; for $\lambda$ a limit ordinal, $J_{\lambda}[x]=\bigcup_{\eta<\lambda} J_{\eta}[x]$.

We say that an ordinal assignment, $\left\{x \mapsto \xi_{x}, x \in \mathbf{R}\right\}$, is reasonable (for the integer $n$ ) (or $n$-reasonable) if it satisfies the following axioms:

(1) For some $\Pi_{n}$-sentence, $\varphi(x), \xi_{x}$ is the least $\xi$ such that $J_{\xi}[x] \vDash \varphi(x)$.

(2) If $y$ is recursive in $x, \xi_{y} \leqslant \xi_{x}$.

(3) $\omega_{1}^{x} \leqslant \xi_{x}$.

It follows from clause (3) that an ordinal assignment is $n$-reasonable only if $n$ is greater than 1 .

We say that $A$ is $\Sigma_{n}\left(J_{\xi_{x}}[x]\right)$ if for some $\Sigma_{n}$ formula $\theta(x)$,

$$
A=\left\{x \in \mathbf{R}: J_{\xi_{x}}[x] \vDash \theta(x)\right\} .
$$

Let $\langle x, y\rangle$ for $x, y \in \mathbf{R}$ be the $z \in \mathbf{R}$ such that $z(2 n)=x(n), z(2 n+1)=y(n)$. We say that $A$ is $\Sigma_{n}\left(J_{\xi_{z}}[z]\right)$ if for some $\Sigma_{n}$ formula $\theta(x)$ and some $y \in \mathbf{R}$,

$$
A=\left\{x:(\exists z \in \mathbf{R})(z=\langle x, y\rangle) \wedge J_{\xi_{z}}[z] \vDash \theta(z)\right\} .
$$

$A$ is $\Delta_{n}\left(J_{\xi_{x}}[x]\right)\left(\operatorname{resp} . \Delta_{n}\left(J_{\xi_{x}}[x]\right)\right)$ if $A$ and $\sim A$ are $\Sigma_{n}\left(J_{\xi_{x}}[x]\right)\left(\operatorname{resp} .\left(\Sigma_{n}\left(J_{\xi_{x}}[x]\right)\right)\right)$.

It is easy to check that if $x \mapsto \xi_{x}$ is $n$-reasonable and $A$ is $\Sigma_{n}\left(J_{\xi_{x}}[x]\right)$, then $A$ is $\Delta_{2}^{1}$. Moreover, every $\Delta_{2}^{1}$ set arises in this way from some $n$-reasonable ordinal assignment.

8.3. Let $\left\{x \mapsto \xi_{x}\right\}$ be $n$-reasonable. Let $A$ be $\Delta_{n}\left(J_{\xi_{x}}[x]\right)$. The assertion that $\left\{x \mapsto \xi_{x}\right\}$ is $n$-reasonable (relative to the formula $\varphi(x)$ used to define the ordinal assignment) is a $\Pi_{3}^{1}$ statement, $\Phi_{1}$ say. The assertion that the $\Sigma_{n}\left(J_{\xi_{x}}[x]\right)$ descriptions of $A, \sim A$ describe complementary sets is a $\Pi_{3}^{1}$ assertion, $\Phi_{2}$.

Let $\mathscr{A}$ be the class of continuous preimages of $A$ (i.e., $B \in \mathscr{A}$ iff $B=F^{-1}[A]$ for some total continuous $F: \mathbf{R} \rightarrow \mathbf{R}$ ).

THEOREM.

$$
\mathrm{ZFC}+\Phi_{1}+\Phi_{2}+\Delta_{n}\left(J_{\xi_{x}}[x]\right)-\mathrm{DET} \vdash \operatorname{Con}\left(\mathrm{ZFC}+\Phi_{1}+\Phi_{2}+\mathscr{A} \text {-DET }\right) .
$$

REMARKS ON THE PROOF. We constructi an ad-hoc class $\Delta$ containing $\mathscr{A}$ to which we can apply the Main Theorem and such that the class $\Gamma$ (as described in the Main Theorem) still lies within $\Delta_{n}\left(J_{\xi_{x}}[x]\right)$. The most delicate point is to insure that $\Delta$ will have the weak scale property.

8.4. Let $\left\{x \mapsto \xi_{x}\right\}$ be $n$-reasonable, as above.

\section{THEOREM.}

$$
\begin{aligned}
\mathrm{ZFC} & +\Phi_{1}+\Phi_{2}+\Sigma_{n}\left(J_{\xi_{x}}[x]\right) \text {-Determinacy } \\
& \vdash \operatorname{Con}\left(\mathrm{ZFC}+\Phi_{1}+\Phi_{2}+\Delta_{n}\left(J_{\xi_{x}}[x]\right) \text {-Determinacy }\right) .
\end{aligned}
$$


REMARKS ON THE PROOF. The obvious set of codes for $\Delta_{n}\left(J_{\xi_{x}}[x]\right)$ is $\Pi_{2}^{1}$. We need an adaptation of the Main Theorem in which the set of $\Delta$-codes is only $\Pi_{2}^{1}$. The key idea is to modify the game so that $I$ can also challenge $\Pi_{2}^{1}$ assertions of the $M$ played by II. We also have to verify that $\Delta_{n}\left(J_{\xi_{x}}[x]\right)$ has the weak scale property. This is done using the scales constructed in the proof of the theorem in 8.3.

\section{REFERENCES}

[G] R. Gostanian, Constructible models of subsystems of ZF, J. Symbolic Logic 45 (1980), 237-250.

[H] L. A. Harrington, Analytic determinacy and $0^{\#}$, J. Symbolic Logic 43 (1978), 685-693.

[Hf] F. Hausdorff, Set theory, 2nd ed., Chelsea, New York, 1962 (translated from Mengenlehre, 3rd ed., 1937).

[J] R. Jensen, The fine structure of the constructible hierarchy, Ann. Math. Logic 4 (1972), 229-308.

[KM] A. Kanamori and M. Magidor, The evolution of large cardinal axioms in set theory, Higher Set Theory (G. H. Müller and D. S. Scott, eds.), Lecture Notes in Math., vol. 669, Springer-Verlag, Berlin and New York, 1978, pp. 99-276.

[Ke1] A. Kechris, The Axiom of Determinacy implies dependent choices in L(R), J. Symbolic Logic (to appear).

[Ke2] __ AD and projective ordinals, Cabal Seminar 76-77 (A. S. Kechris and Y. N. Moschovakis, eds.), Lecture Notes in Math., vol. 689, Springer-Verlag, Berlin and New York, 1978, pp. 91-132.

[KeM] A. Kechris and Y. Moschovakis, Notes on the theory of scales, Cabal Seminar 76-77 (A. S. Kechris and Y. N. Moschovakis, eds.), Lecture Notes in Math., vol. 689, Springer-Verlag, Berlin and New York, 1978, pp. 1-54.

[KeW] A. Kechris and H. Woodin, The equivalence of partition properties and determinacy, Proc. Nat. Acad. Sci. U.S.A. 80 (1983), 1783-1786.

[Ma1] D. A. Martin, Measurable cardinals and analytic games, Fund. Math. 66 (1970), 287-291.

[Ma2] , Borel and projective games (to appear).

[Ma3] _ Infinite games, Proc. Internat. Congr. Math., Helsinki, 1978, pp. 269-273.

[Ma4] _ , $\Delta_{2 n}^{1}$ determinacy implies $\Sigma_{2 n}^{1}$ determinacy, mimeographed notes, 1973.

[Ma5] _ , $\Delta_{2 n}^{1}$ determinacy $\Rightarrow \Delta_{2 n+1}^{1}$ determinacy, mimeographed notes, May 1974.

[MMS] D. A. Martin, Y. N. Moschovakis and J. R. Steel, The extent of definable scales, Bull. Amer. Math. Soc. (N.S.) 6 (1982), 435-440.

[MS] D. A. Martin and R. M. Solovay, A basis theorem for $\Sigma_{3}^{1}$ sets of reals, Ann. of Math. (2) 89 (1969), $138-160$.

[MSt] D. A. Martin and J. R. Steel, The extent of scales in L(R), Cabal Seminar 79-81 (A. S. Kechris, D. A. Martin and Y. N. Moschovakis, eds.), Lecture Notes in Math., vol. 1019, Springer-Verlag, Berlin and New York, 1983, pp. 86-97.

[Mi1] W. Mitchell, Sets constructible from sequences of ultrafilters, J. Symbolic Logic 39 (1974), 57-66.

[Mi2] __ Hypermeasurable cardinals, Proc. Logic Colloq. 78 (M. Boffa, D. Van Dalen and K. McAloon, eds.), North-Holland, Amsterdam, 1979, pp. 303-317.

[Mo1] Y. N. Moschovakis, Descriptive set theory, North-Holland, Amsterdam, 1980.

[Mo2] _ Scales on coinductive sets, Cabal Seminar 79-81 (A. S. Kechris, D. A. Martin and Y. N. Moschovakis, eds.), Lecture Notes in Math., vol. 1019, Springer-Verlag, Berlin and New York, 1983, pp. $77-86$.

[Si] J. Simms, Ph.D. Thesis, Rockefeller University, 1979.

[St1] J. R. Steel, Determinacy in the Mitchell models, Ann. Math. Logic 22 (1982), 109-125.

[St2] Scales on $\Sigma_{1}^{1}$ sets, Cabal Seminar 79-81 (A. S. Kechris, D. A. Martin and Y. N. Moschovakis, eds.), Lecture Notes in Math., vol. 1019, Springer-Verlag, Berlin and New York, 1983, pp. $72-77$.

Department of Mathematics, California institute of Technology, Pasadena, California 91125

Department of Mathematics, University of California, Berkeley, California 94720 